\title{
Design of Perfect-Reconstruction Nonuniform Recombination Filter Banks With Flexible Rational Sampling Factors
}

\author{
X. M. Xie, S. C. Chan, and T. I. Yuk
}

\begin{abstract}
This paper studies the design of a class of perfect-reconstruction (PR) nonuniform filter banks (FBs) called recombination nonuniform FBs (RNFBs). They are constructed by merging subbands in a uniform FB with sets of transmultiplexers (TMUXs). It generalizes the RNFBs previously proposed by the authors to allow more general choice of the sampling factors. The spectral inversion and spurious response suppression problems of these new RNFBs using cosine modulation are analyzed, and a simple design method based on a matching condition is proposed. It is also found that the FB and the TMUX in the recombination structure can be designed separately to satisfy the matching condition. In addition, real-time adaptive merging of the channels to provide dynamic nonuniform frequency partitioning is feasible. Another advantage of the RNFBs is that the recombination and processing of the subband signal can be done at the decimated domain of the uniform FB, which greatly reduces its implementation complexity. Design examples show that high quality nonuniform PR FBs with low implementation complexity and variable time-frequency resolution can be obtained by the proposed method.
\end{abstract}

Index Terms-Cosine modulated filter banks (CSFBs), dynamic recombination, FBs, perfect reconstruction, rational sampling factors.

\section{INTRODUCTION}

$\mathbf{P}$ ERFECT-RECONSTRUCTION (PR) quadrature mirror filter (QMF) bank has important applications in speech, audio, image, and array signal processing. They can also be used to generate orthogonal as well as biorthogonal wavelet bases provided that the filter banks (FBs) satisfy certain regularity conditions. The theory of PR $M$-channel maximally decimated uniform FBs has been extensively studied in [1], [13], [19]-[22]. In some applications such as audio coding, spectral analysis, and subband adaptive filtering, nonuniform frequency partitioning may be used to better exploit the signal characteristics. For example, in audio coding, it is advantageous to employ PR nonuniform FBs, which approximate the nonuniform frequency sensitivities of the human auditory system (the Bark scale) [2]. The theory and design of nonuniform FBs were previously studied in [2]-[11], [16], [18], [23], [24]. Interested readers are referred to the review in [8] and the references therein. A novel $\mathcal{H}_{\infty}$ approach [18]

Manuscript received December 24, 2003; revised December 5, 2004. This paper was recommended by Associate Editor M. G. Strintzis.

The authors are with the Department of Electrical and Electronic Engineering, University of Hong Kong, Hong Kong (e-mail: scchan@eee.hku.hk).

Digital Object Identifier 10.1109/TCSI.2005.852009 was proposed to determine the synthesis filters from a given set of analysis filters. The system is pseudo-PR and its PR violation is of the order of $10^{-3}$. Another useful approach for designing nonuniform FBs is the time domain representation proposed by Nayebi et al. [10]. Pseudo-PR nonuniform FBs based on cosine-modulated filter banks (CMFBs) were also studied in [3], [11], [16], [23]. Like their uniform counterparts, nonuniform CMFBs have the potential to offer low design and implementation complexities. In [23], a class of pseudo-PR nonuniform FBs were obtained by combining directly some of the decimated branches of CMFBs. However, since the combining is performed before the decimators, the arithmetic complexity is rather high. Fig. 1 shows a two-stage structure for nonuniform FB that was originally proposed by Cox [3], where certain channels in an $M$-channel uniform FB are combined or merged together using the synthesis filters of transmultiplexers (TMUXs) with smaller channel numbers. Because of the merging operation, we shall call this method the indirect or recombination (merging) method. In Cox's original work, the analysis and synthesis filters of the FB and TMUXs are derived from pseudo-quadrature mirror filters, which are not PR. In [4], a comprehensive theory and detailed classification of nonuniform FBs were given. In contrast, a direct structure as shown in Fig. 2 was proposed which has better control and freedom over the indirect method. This is because the indirect method-based RNFBs do not generally lead to linear time-invariant (LTI) analysis filters and, as a result, the two stages of the RNFBs are usually designed separately. Furthermore, the direct method allows one to have complete control over the desired frequency characteristics of the LTI analysis filters $\left(H_{0}, H_{1}, \cdots, H_{L-1}\right)$. Recently, the authors showed that [5], [6], [17] it is possible to construct PR nonuniform FBs from PR uniform FBs and TMUXs in the two-stage or recombination structure. The analysis and synthesis filters of these PR RNFBs are in general linear periodically time varying (LPTV), though they satisfy the PR condition. Therefore, the design of such LPTV systems is in general rather involved. More recently, it has been shown [5], [6], [14] that if the numbers of channels in the original FB and the recombination TMUXs are coprime, then the analysis and synthesis filters can either be represented as LTI systems or the cascade of certain LTI systems with the modulation sequence $(-1)^{n}$. As a result, the design complexity of the RNFBs can be reduced considerably. The introduction of the modulation sequence $(-1)^{n}$ is to overcome the spectral inversion problem, which is inherent in general nonuniform FBs. It was also shown that, if efficient filter structures, such as the 


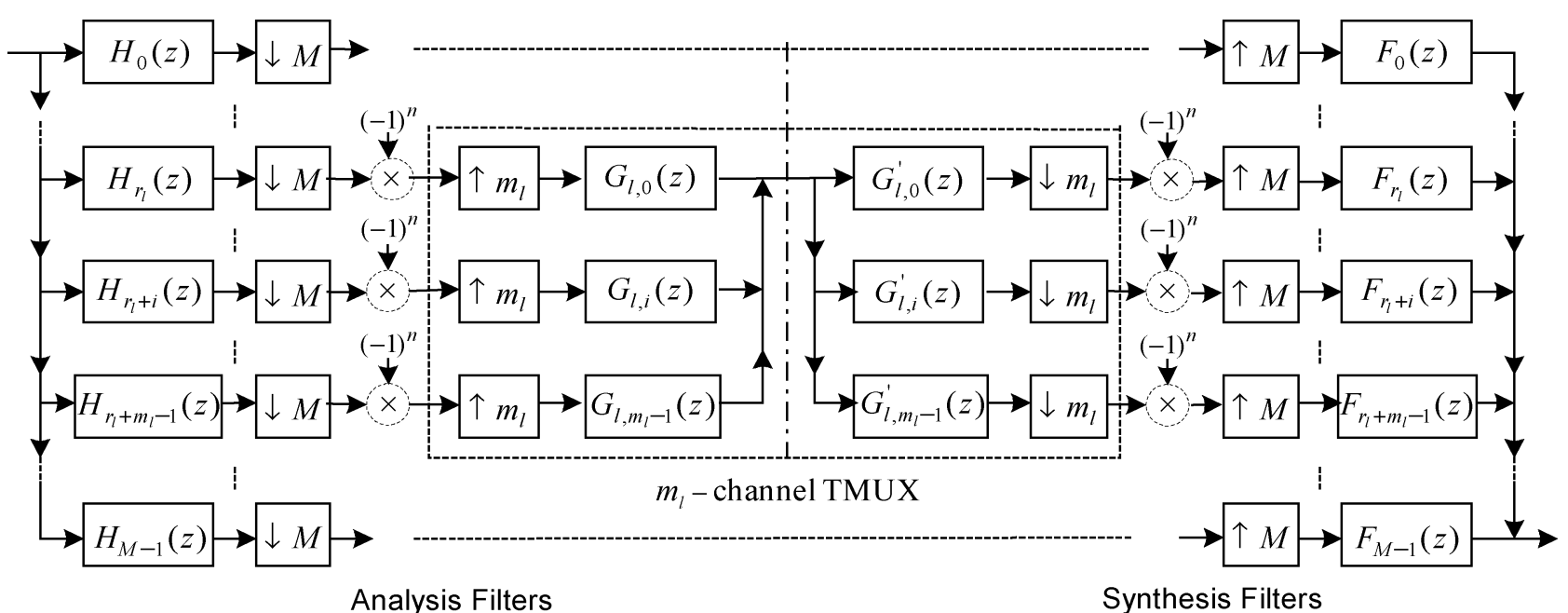

Fig. 1. Structure of RNFB.

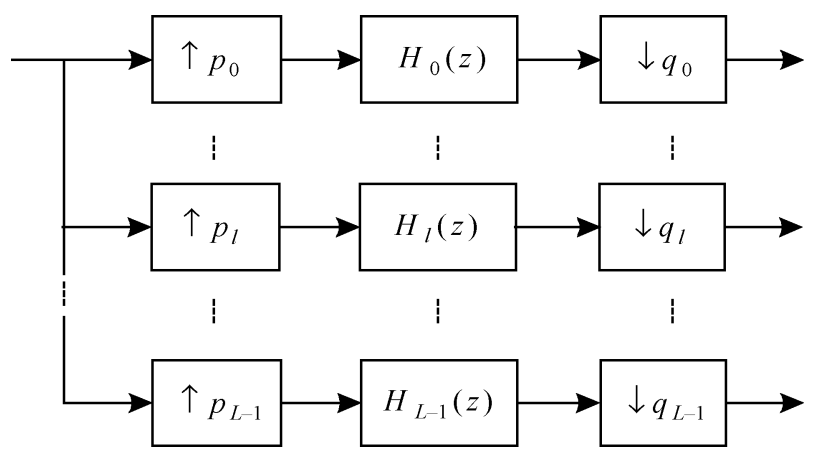

Fig. 2. Direct structure in [4]. $\left(p_{l}, q_{l}\right)$ are coprime, and $\left(\sum_{l=0}^{L-1}\left(p_{l} / q_{l}\right)=1\right)$.

CMFBs, are used as original uniform FBs and recombination TMUXs, the design and implementation complexities of the nonuniform FBs will be greatly reduced.

In this paper, we consider the more general case where the channel numbers of the FB and the TMUXs are not coprime to each other, thereby offering more flexibility in choosing the sampling factors. Like their coprime counterparts, the major difficulty in designing such RNFBs is the suppression of spurious frequency components resulting from the overlapping of frequency-shifted responses of the analysis filters in the uniform FB and recombination TMUXs. In the coprime case, this problem for the RN-CMFB is easier to analyze, because the analysis filters admit an LTI representation. It was found that [5], [6] by imposing a simple matching condition on the filter length and transition bandwidth of the prototype filters, the spurious response can be effectively suppressed. Moreover, the original uniform FB and the recombination TMUXs could be designed separately, which greatly simplifies the design procedure. In this paper, the origin of these spurious responses for general $\mathrm{RN}$-CMFBs is analyzed. It is found that if the prototype filters of the FB and the TMUXs satisfy a similar matching condition as the coprime case, then the undesirable components of the analysis filters can also be suppressed. Comparing to the pseudo-nonuniform CMFB in [16] where the direct structure was employed as shown in Fig. 2, both the PR violation $\left(10^{-3}\right.$ versus $10^{-15}$ ) and implementation complexity of the proposed
RN-CMFB is considerably lower, at the expense of lower stopband attenuation. This is because the recombination and processing of the subband signal can be done at the decimated domain of the uniform FB, which greatly reduces the implementation complexity and power dissipation for high-speed hardware implementation. On the other hand, since the outputs of the analysis filters in [23] are combined before the decimators, it has a higher complexity than the proposed approach. Moreover, as the original FB and merging TMUXs can be designed separately, real-time adaptive merging of channels to provide dynamically nonuniform frequency partitioning is feasible in the proposed structure [17]. Several design examples will be given to demonstrate this principle and the usefulness of the proposed RN-CMFBs. It is found that the RNFBs are useful in improving the time resolution of the original uniform FB and isolating signal components with different bandwidths by adaptively recombining selected number of subchannels using sets of predesigned TMUXs. It can be also combined with traditional tree-structured FBs to provide a wide range of different adaptive configurations. Furthermore, the proposed nonuniform CMFBs can also be used to realize nonuniform TMUXs, which are very useful in software radio receivers and other communication applications. The rest of this paper is organized as follows. The general principle of the PR RNFB is described in Section II. Section III is devoted to the analysis of the spurious response and the derivation of the matching condition for its suppression in RN-CMFB. The design procedure and examples are shown in Section IV. Finally, conclusions are given in Section V.

\section{PR RECOMBINATION NONUNIFORM FILTER BANKS (RNFBS)}

Referring to the $L$-band RNFB shown in Fig. 1. It can be seen that the $m_{l}$ consecutive channels starting at the $r_{l}$ th channel in an $M$-channel uniform FB are combined or merged using the synthesis filters $G_{l, i}(z)$ of an $m_{l}$-channel TMUX, where $l=0,1, \cdots, L-1$ and $i=0,1, \cdots, m_{l}-1$. This produces the $l$ th subband output of the nonuniform FB. In the synthesis section, the analysis filters $G_{l, i}^{\prime}(z)$ of the $m_{l}$-channel FB is used to regenerate the signals in these subchannels. It 
can be seen that the merging part, $G_{l, i}(z)$, and the decomposing part, $G_{l, i}^{\prime}(z)$, enclosed by the dotted lines in Fig. 1, constitute a TMUX. If the $M$-channel uniform FB and the TMUX are PR, then the whole system will be PR, provided that the delay of the TMUX is properly compensated in other bands. The PR condition of uniform TMUX is closely related to that of PR uniform FB [15], and their design procedures are similar to each other. More precisely, the PR condition of a uniform TMUX is identical to that of a 1-skewed PR FB. Let $\left\{H_{k}(z), F_{k}(z)\right\}, k=0,1, \cdots, M-1$, be the analysis and synthesis filters of a PR uniform FB. A 1-skewed PR FB can be derived from this PR FB by choosing its analysis and synthesis filters as $\left\{H_{k}^{\prime}(z), F_{k}^{\prime}(z)\right\}$, where $H_{k}^{\prime}(z)=H_{k}(z)$ and $F_{k}^{\prime}(z)=z^{-1} F_{k}(z)$. Therefore, if $G_{l, i}(z)$ and $G_{l, i}^{\prime}(z)$ in Fig. 1 are the synthesis and analysis filters of an $m_{l}$-channel 1-skewed PR FB, then they constitute a PR TMUX. Since different TMUXs might have different delays, depending on the filters $G_{l, i}(z)$ and $G_{l, i}^{\prime}(z)$, appropriate delay has to be inserted in each subband to ensure that the overall system is PR. Because of this structural property, it is relatively simple to obtain PR nonuniform FB from PR uniform FBs using the recombination approach.

As mentioned in Section I, the RNFBs do not generally possess equivalent LTI filter representations like the direct structure in [4]. The analysis and synthesis filters are in general LPTV, though the entire system is PR. To gain more insight into the problem, let us consider the discrete-time Fourier transform (DTFT) of the $l$ th merged output in the RNFB. Assume that the $m_{l}$ channels starting from the $r_{l}$ th channel of the $M$-channel uniform FB are merged by the synthesis filters, $G_{l, i}(z)$, of an $m_{l}$-channel TMUX. The output of the merged channels is

$$
\begin{aligned}
Y_{l}\left(e^{j \omega}\right)=\frac{1}{M} & \sum_{i=0}^{m_{l}-1} G_{l, i}\left(e^{j \omega}\right) \\
& \times \sum_{v=0}^{M-1} H_{r_{l}+i}\left(e^{j \frac{m_{l} \omega-2 \pi v}{M}}\right) X\left(e^{j \frac{m_{l} \omega-2 \pi v}{M}}\right)
\end{aligned}
$$

where $X\left(e^{j \omega}\right)$ and $Y_{l}\left(e^{j \omega}\right)$ are, respectively, the DTFT of the input and the $l$ th nonuniform subband output. $Y_{l}\left(e^{j \omega}\right)$ contains the desired signal component generated by an ordinary LTI analysis filter and the aliasing components generated from the decimation operation. For ideal analysis filters with infinite sharp cutoff, all the aliasing terms will be zero. For practical filters with finite length, we would expect the aliasing terms to be small when the original FB and recombination TMUXs have sufficiently large stopband attenuation.

Another problem frequently encountered in the design of nonuniform $\mathrm{FB}$ is the spectral inversion problem. In the context of RNFBs, this problem occurs when certain outputs of the $M$-channel uniform FB are merged. More precisely, for ideal filters, $G_{l, i}\left(e^{j \omega}\right)$ should have the same rectangular shape and location as $H_{r_{l}+i}\left(e^{j m_{l} \omega / M}\right)$, and $(1 / M) \sum_{i=0}^{m_{l}-1} G_{l, i}\left(e^{j \omega}\right) H_{r_{l}+i}\left(e^{j\left(m_{l} \omega / M\right)}\right)$ should cover the whole frequency range from 0 to $\pi$. Under this situation, the frequency support of $G_{l, i}(z)$ and $H_{r_{l}+i}(z)$ are $\left(\left(\pi / m_{l}\right) i,\left(\pi / m_{l}\right)(i+1)\right)$ and $\left((\pi / M)\left(r_{l}+i\right),(\pi / M)\left(r_{l}+\right.\right.$

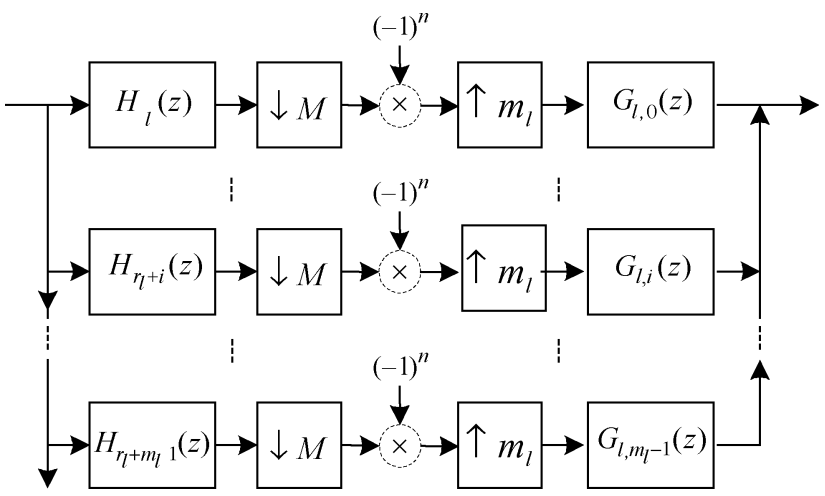

(a)

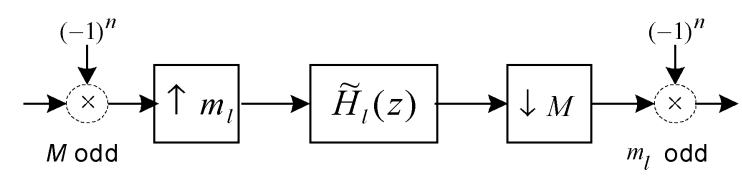

(b)

Fig. 3. (a) The $l$ th branch of the analysis section in the RNFB, (b) its equivalent structure when $M$ and $m_{l}$ are coprime.

$i+1)$ ), respectively. After decimation and interpolation, the frequency range of $H_{r_{l}+i}\left(e^{j m_{l} \omega / M}\right)$ becomes $\left(\left(\pi / m_{l}\right)\left(r_{l}+i\right) \pm\left(2 \pi v / m_{l}\right),\left(\pi / m_{l}\right)\left(r_{l}+i+1\right) \pm\left(2 \pi v / m_{l}\right)\right.$. For $G_{l, i}\left(e^{j \omega}\right)$ and $H_{r_{l}+i}\left(e^{j m_{l} \omega / M}\right)$ to have the same location, we must have: $r_{l}+i \pm 2 v=i$. In other words, $r_{l}$ must be even, and even for ideal filters, there will be spectral inversion in the merged subbands, if $r_{l}$ is odd. This problem, which is an inherent problem of nonuniform FBs, was also mentioned in [4] but no solution was offered. Careful examination reveals that if $r_{l}$ is odd, then the output spectrum is merely inverted. This can be corrected by multiplying the sequence $\left\{(-1)^{n}\right\}$ to the input of the TMUX. This method to handle the spectral inversion problem was first proposed by Cox in [3] for designing RNFBs using the pseudo-QMF banks. Recently, the authors have shown that when $M$ and $m_{l}$ are coprime, then the downsamplers and the upsamplers in the RNFB can be interchanged to obtain LTI analysis filters [5]. More precisely, using the noble identity [13], $H_{r_{l}+i}(z)$ and $G_{l, i}(z)$ can be moved, respectively, across the interpolators $\left(m_{l}\right)$ and the decimators $(M)$ to form the cascaded filters $H_{r_{l}+i}\left(z^{m_{l}}\right)$ and $G_{l, i}\left(z^{M}\right)$. As a result, the $m_{l}$ branches can be represented as an equivalent LTI filter given by $\widetilde{H}_{l}(z)=\sum_{i=0}^{m_{l}-1} H_{r_{l}+i}\left(z^{m_{l}}\right) G_{l, i}\left(z^{M}\right)$ [5]. In order to overcome the problem of spectral inversion when the starting index $r_{l}$ is odd, the outputs of the merged channels should be multiplied by the modulation sequence $\left\{(-1)^{n}\right\}$ [3]-[5]. It is also possible to obtain an equivalent representation having an LTI filter cascading with the modulation $\left\{(-1)^{n}\right\}$ as shown in Fig. 3 [14], where $\widetilde{H}_{l}(z)=\sum_{i=0}^{m_{l}-1} H_{r_{l}+i}\left(z^{m_{l}}\right) G_{l, i}\left(-z^{M}\right)$ for odd $m_{l}$ and $\widetilde{H}_{l}(z)=\sum_{i=0}^{m_{l}-1} H_{r_{l}+i}\left(-z^{m_{l}}\right) G_{l, i}\left(z^{M}\right)$ for odd $M$. The modulated consequence $\left\{(-1)^{n}\right\}$ is in the left side when $m_{l}$ is odd and in the right side when $M$ is odd. The frequency responses of the resulting LTI analysis filters can then be minimized by means of some performance measures, such as the stopband attenuation and passband ripples. The LTI representation is very important to software radio base station applications because the received signal might spread over 


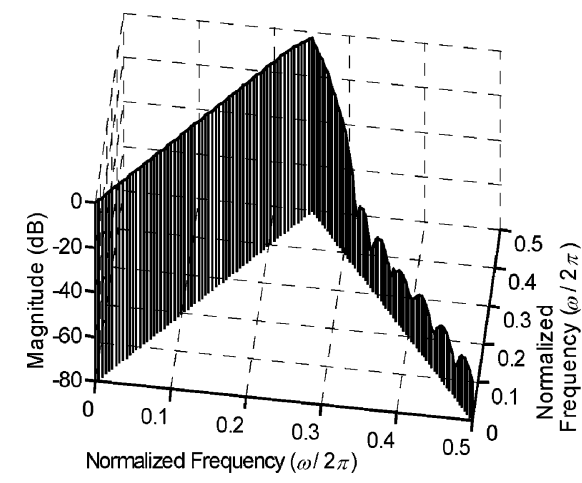

(a)

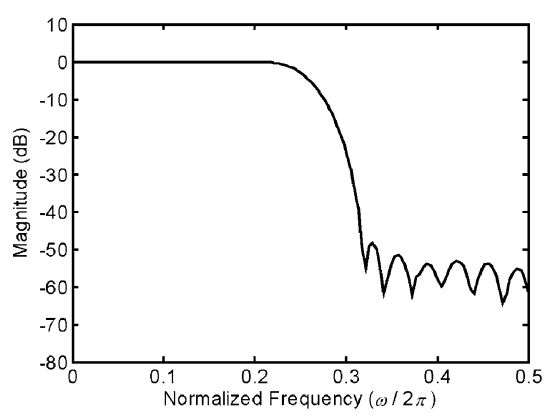

(b)

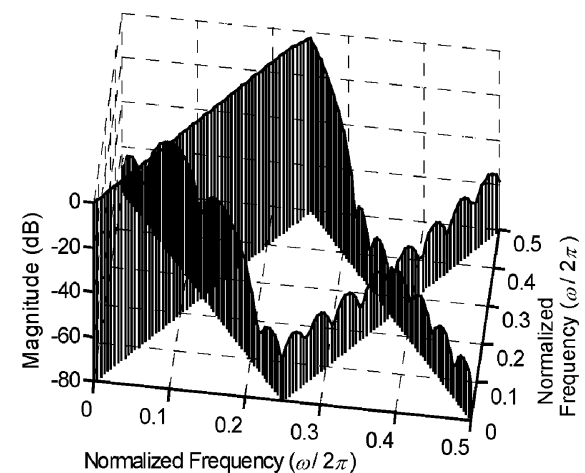

(c)

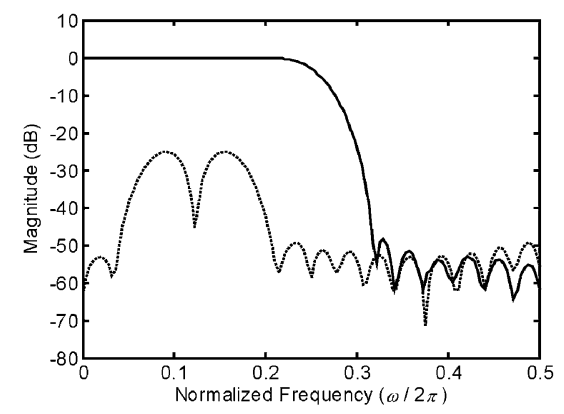

(d)

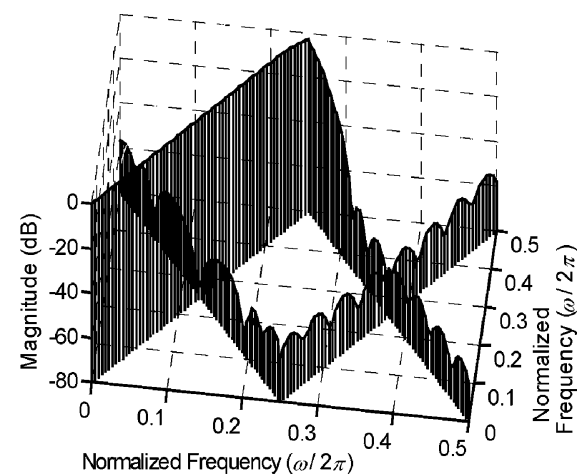

(e)

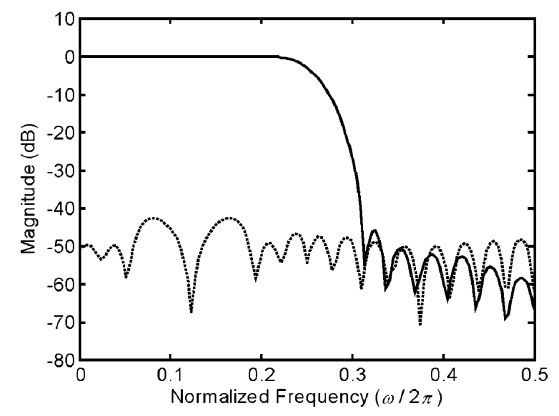

(f)

Fig. 4. Responses of the LTI filter-based rate-1/2 decimator and the first analysis filter of the RN-CMFB with sampling factor (2/4,1/4,1/4). (a) Bifrequency responses of the LTI filter. (b) Its projection. (c) Bifrequency responses of the LPTV filter-matching condition not satisfied. (d) Their projections (with additional aliasing term). (e) Bifrequency responses of the LPTV filter-matching condition satisfied. (f) Their projections.

several subbands, which are to be merged adaptively (please refer to the design examples for more details). If the merged analysis filter is LTI, then the merged output is equivalent to passing the received signal through the given LTI filter and decimating it to the output rate. As a result, any distortion can be precisely controlled despite the presence of the up-and down-sampling operations. Unfortunately, when $M$ and $m_{l}$ are not coprime, the decimators and interpolators cannot be interchanged, and the analysis and synthesis filters are LPTV. This calls for a new method to minimize the additional aliasing terms as mentioned in (1). To avoid complicated nonlinear optimization in the bifrequency plane, we propose a simple matching condition for the prototype filters in RN-CMFBs so that they can be designed separately to yield RN-CMFBs with good frequency characteristics.

To better understand this problem, let us consider a simple RNFB with sampling factors $(2 / 4,1 / 4,1 / 4)$, where the first two channels of an length-40 4-channel uniform CMFB are merged by the synthesis filters of an length-20 2-channel CMFB-based TMUX yielding an equivalent subband with sampling factor $2 / 4$. Since the channel numbers of the original FB and the recombination TMUX are not coprime, the analysis filter is LPTV. The bifrequency responses of the LPTV analysis filter are illustrated in Fig. 4, where the amplitudes and phases of all the frequency components are generated by inputting a sinusoidal signal at certain frequency with unit amplitude and zero phase. In Fig. 4(a), the bifrequency response of an ideal LTI analysis filter with cutoff frequency at $\pi / 2$ is shown. Fig. 4(b) shows its projection. Note, the decimation causes the input signal to be aliased to another frequency when the input frequency is larger than $\pi / 2$. Fig. $4(\mathrm{c})$ shows the bifrequency responses of a practical LPTV analysis filter. The additional V-shaped responses are the aliasing term generated by the down-and-up sampling of the LPTV filter. The projections of these components are shown in Fig. 4(d), containing the LTI portion of this LPTV filter and the additional aliasing term. It can also be seen that there are considerable aliasing components, called the spurious response, around $\pi / 4^{1}$ due to the mismatching of the CMFBs. From the discussion in Section III, it will be found that this spurious response can be suppressed if the prototype filters $H\left(e^{j \omega}\right)$ and $G_{l}\left(e^{j \omega}\right)$ satisfy a matching condition. This is illustrated in Fig. 4(e) and (f), where the spurious response is suppressed by imposing this matching condition. The origin of this spurious response and the matching condition will be analyzed in the following section.

\section{PR RECOMBINATION NONUNIFORM CMFBS (RN-CMFBS)}

\section{A. $C M F B$}

In CMFBs, the analysis and synthesis filters are frequency shifted versions of a prototype filter. Without loss of generality, the type-IV discrete cosine transform (DCT)-based CMFB proposed in [12] is considered. The analysis filters $h_{k}(n)$ and synthesis filters $f_{k}(n)$ are given by

$$
h_{k}(n)=2 h(n) \cos \left[\frac{(2 k+1) \pi}{2 M}\left(n-\frac{N-1}{2}\right)+(-1)^{k} \frac{\pi}{4}\right]
$$

${ }^{1}$ For the coprime case, the analysis filters are LTI. Similar spurious response will also occur, and it will appear at the stopband of the filter. 


$$
\begin{gathered}
f_{k}(n)=2 h(n) \cos \left[\frac{(2 k+1) \pi}{2 M}\left(n-\frac{N-1}{2}\right)-(-1)^{k} \frac{\pi}{4}\right] \\
k=0,1, \cdots, M-1, n=0,1, \cdots, N-1
\end{gathered}
$$

where $h(n)$ is the impulse response of the prototype filter and $N$ is the filter length. For simplicity, we shall consider the case of $N=2 m M$. Let $H(z)=\sum_{q=0}^{2 M-1} z^{-q} P_{q}\left(z^{2 M}\right)$ be the type-I polyphase decomposition [13] of the prototype filter, it can be shown [25], [26] that the PR conditions of the CMFB are given by

$$
\begin{array}{r}
P_{k}(z) P_{2 M-k-1}(z)+P_{M+k}(z) P_{M-k-1}(z)=\beta \cdot z^{-\sigma}, \\
k=0,1, \cdots, M-1
\end{array}
$$

where $\beta$ is a nonzero constant and $\sigma$ is a positive integer. Since the analysis and synthesis filters are frequency shifted versions of the prototype filter, it is only necessary to minimize the passband and stopband ripples of the prototype filter. More precisely, the design problem can be formulated as the following constrained optimization problem

$$
\min _{h} \Phi=\alpha \int_{0}^{\omega_{p}}\left|1-H\left(e^{j \omega}\right)\right|^{2} d \omega+(1-\alpha) \int_{\omega_{s}}^{\pi}\left|H\left(e^{j \omega}\right)\right|^{2} d \omega
$$

subject to the PR constraints in(3)

where $\alpha$ is a weighting constant between 0 and $1 ; \omega_{p}$ and $\omega_{s}$ are the passband and stopband cutoff frequencies. The parameters to be optimized are the impulse response coefficients of the prototype filter. Therefore, the number of optimization variables is largely reduced as compared to a general PR FB. For simplicity, we only consider orthogonal CMFBs in this paper, and (3) is reduced to

$P_{k}(z) \widetilde{P}_{k}(z)+P_{M+k}(z) \widetilde{P}_{M+k}(z)=\beta, \quad k=0,1, \cdots, M-1$

where $\widetilde{P}(z)=\widetilde{P}_{*}\left(z^{-1}\right)$, * denotes conjugation of coefficients, and $\alpha$ in (4) can then be chosen as zero. The constrained optimization involved in (4) can be solved using the DNCONF subroutine in the International Mathematics and Statistics (IMSL) library.

\section{B. The Matching Condition for Spurious Response Suppression}

In the RNFB, the DTFTs of the $m_{l}$ analysis filters of the $M$-channel CMFB, starting from $r_{l}$, and the synthesis filters of the $m_{l}$-channel CMFB are given by ${ }^{2}$

$$
\begin{aligned}
H_{r_{l}+i}(\omega)= & \frac{1}{2} e^{j \theta_{r_{l}+i} H\left(\omega-\omega_{r_{l}+i}\right)} \\
& +\frac{1}{2} e^{-j \theta_{r_{l}+i}} H\left(\omega+\omega_{r_{l}+i}\right) \\
G_{l, i \_F B}(\omega)= & \frac{1}{2} e^{j \xi_{l, i}} G_{l}\left(\omega-\eta_{l, i}\right)+\frac{1}{2} e^{-j \xi_{l, i}} G_{l}\left(\omega+\eta_{l, i}\right) \\
& i=0,1, \cdots m_{1}-1
\end{aligned}
$$

${ }^{2}$ For simplicity, we denote the frequency response by $H(\omega)$ instead of $H\left(e^{j \omega}\right)$, and vice versa. where $\omega_{r_{l}+i}=\left(2\left(r_{l}+i\right)+1\right) \pi /(2 M), \theta_{r_{l}+i}=-\left(2\left(r_{l}+i\right)+\right.$ 1) $N_{M} \pi /(4 M)+(-1)^{\left(r_{l}+i\right)} \pi / 4, \eta_{l, i}=(2 i+1) \pi /\left(2 m_{l}\right)$ and $\xi_{l, i}=-(2 i+1) N_{m_{l}} \pi /\left(4 m_{l}\right)-(-1)^{i} \pi / 4 . H(\omega)$ and $G_{l}(\omega)$ are, respectively, the prototype filters of the $M$ - and $m_{l}$-channel CMFBs. To simplify the analysis, we assume that they are bandlimited to the region $|\omega| \leq \pi / M$ and $|\omega| \leq \pi / m_{l}$, respectively. Therefore, major aliasing components will result when the transition bands of adjacent modulated filter response overlap each other. $N_{M}$ and $N_{m_{l}}$ denote the lengths of $H(\omega)$ and $G_{l}(\omega)$, respectively. Here, we only examine the case where the starting index $r_{l}$ is even. The odd value case is rather similar and the details are summarized in the Appendix. First of all, rewrite (1) as

$$
Y_{l}(\omega)=\frac{1}{M} \sum_{v=0}^{M-1} A_{v}(\omega) X\left(\frac{m_{l} \omega-2 \pi v}{M}\right)
$$

where

$$
\begin{aligned}
A_{v}(\omega)=\sum_{i=0}^{m_{l}-1} G_{l, i}(\omega) H_{r_{l}+i}\left(\frac{m_{l} \omega-2 \pi v}{M}\right) & \\
& v=0,1, \cdots, M-1 .
\end{aligned}
$$

Note, $Y_{l}(\omega)$ has a period of $2 \pi M / m_{l}$. We would like to design $A_{v}(\omega)$ so that only the appropriate frequency components of $X(\omega)$ are extracted. Our goal here is to suppress the unwanted aliasing components and ensure that the analysis filter has a good filter quality. As mentioned above, the synthesis filters $G_{l, i}(\omega)$ of the PR TMUX are derived from a 1-skewed PR FB with prototype filter $G_{l}(\omega)$ and $G_{l, i}(\omega)=e^{-j \omega} G_{l, i_{-} F B}(\omega)$. For notation simplicity, define $\bar{H}_{r_{l}+i}(\omega)=H_{r_{l}+i}\left(\left(m_{l} / M\right) \omega\right)$. Thus, (8) can be rewritten as

$$
\begin{aligned}
A_{v}(\omega)=\sum_{i=0}^{m_{l}-1} e^{-j \omega} G_{l, i_{-} F B}(\omega) \bar{H}_{r_{l}+i} & \left(\omega-\frac{2 \pi v}{m_{l}}\right), \\
v & =0,1, \cdots, M-1 .
\end{aligned}
$$

From (5) and (6), $\bar{H}_{r_{l}+i}(\omega)$ and $G_{l, i_{-} F B}(\omega)$ can further be written as

$$
\begin{aligned}
\bar{H}_{r_{l}+i}(\omega) & =\frac{1}{2} e^{j \theta_{r_{l}+i}} \bar{H}_{r_{l}+i}^{+}(\omega)+\frac{1}{2} e^{-j \theta_{r_{l}+i}} \bar{H}_{r_{l}+i}^{-}(\omega) \\
G_{l, i_{-} F B}(\omega) & =\frac{1}{2} e^{j \xi_{l, i}} G_{l, i}^{+}(\omega)+\frac{1}{2} e^{-j \xi_{l, i}} G_{l, i}^{-}(\omega)
\end{aligned}
$$

where

$$
\begin{aligned}
\bar{H}_{r_{l}+i}^{+}(\omega) & =\bar{H}\left(\omega-\frac{M}{m_{l}} \omega_{r_{l}+i}\right) \\
\bar{H}_{r_{l}+i}^{-}(\omega) & =\bar{H}\left(\omega+\frac{M}{m_{l}} \omega_{r_{l}+i}\right) \\
G_{l, i}^{+}(\omega) & =G_{l}\left(\omega-\eta_{l, i}\right) \\
G_{l, i}^{-}(\omega) & =G_{l}\left(\omega-\eta_{l, i}\right) .
\end{aligned}
$$

Substituting (10) and (11) into (9), one gets

$$
\begin{aligned}
& A_{v}(\omega)=e^{-j \omega} \sum_{i=0}^{m_{l}-1}( \frac{1}{2} e^{j \theta_{r_{l}+i}} \bar{H}_{r_{l}+i}^{+}\left(\omega-\frac{2 \pi v}{m_{l}}\right) \\
&\left.+\frac{1}{2} e^{-j \theta_{r_{l}+i}} \bar{H}_{r_{l}+i}^{-}\left(\omega-\frac{2 \pi v}{m_{l}}\right)\right)
\end{aligned}
$$




$$
\begin{aligned}
& \times\left(\frac{1}{2} e^{j \xi_{l, i}} G_{l, i}^{+}(\omega)+\frac{1}{2} e^{-j \xi_{l, i}} G_{l, i}^{-}(\omega)\right) \\
= & e^{-j \omega} \sum_{j=1}^{4} D_{j}^{(v)}(\omega)
\end{aligned}
$$

where

$$
\begin{aligned}
& D_{1}^{(v)}(\omega)=\frac{1}{4} \sum_{i=0}^{m_{l}-1} e^{j\left(\theta_{r_{l}+i}+\xi_{l, i}\right)} \bar{H}_{r_{l}+i}^{+}\left(\omega-\frac{2 \pi v}{m_{l}}\right) G_{l, i}^{+}(\omega) \\
& D_{2}^{(v)}(\omega)=\frac{1}{4} \sum_{i=0}^{m_{l}-1} e^{j\left(\theta_{r_{l}+i}-\xi_{l, i}\right)} \bar{H}_{r_{l}+i}^{+}\left(\omega-\frac{2 \pi v}{m_{l}}\right) G_{l, i}^{-}(\omega) \\
& D_{3}^{(v)}(\omega)=\frac{1}{4} \sum_{i=0}^{m_{l}-1} e^{-j\left(\theta_{r_{l}+i}-\xi_{l, i}\right)} \bar{H}_{r_{l}+i}^{-}\left(\omega-\frac{2 \pi v}{m_{l}}\right) G_{l, i}^{+}(\omega) \\
& D_{4}^{(v)}(\omega)=\frac{1}{4} \sum_{i=0}^{m_{l}-1} e^{-j\left(\theta_{r_{l}+i}+\xi_{l, i}\right)} \bar{H}_{r_{l}+i}^{-}\left(\omega-\frac{2 \pi v}{m_{l}}\right) G_{l, i}^{-}(\omega) .
\end{aligned}
$$

It will be shown that $D_{1}^{(v)}(\omega)$ and $D_{4}^{(v)}(\omega)$ together constitute the complete desired spectrum with a period of $2 \pi$. For practical filters with finite support, transition bands of these frequency-shifted copies might overlap to yield undesirable or spurious responses and all of them are coming from $D_{2}^{(v)}(\omega)$. The matching condition to be introduced in (32) and (33) will help us to suppress the unwanted response in $D_{2}^{(v)}(\omega)$.

Next, we shall take a closer look at the four terms $D_{j}^{(v)}(\omega)$, $j=1,2,3,4$. We only need to consider $D_{1}^{(v)}(\omega)$ and $D_{2}^{(v)}(\omega)$, since $D_{3}^{(v)}(\omega)$ and $D_{4}^{(v)}(\omega)$ are their conjugates. Before that, it is necessary to determine the supports of $\bar{H}_{r_{l}+i}(\omega)$ and $G_{l, i}(\omega)$.

Supports of $\bar{H}_{r_{l}+i}(\omega)$ and $G_{l, i}(\omega)$ : First of all, denote the frequency support of a function $Q(\omega)$ as $\operatorname{Supp}(Q)$. If $\omega \notin$ $\operatorname{Supp}(Q)$, then $Q(\omega)=0$. Therefore, for $H(\omega)$ and $G_{l}(\omega)$, we have $\operatorname{Supp}(H)=[2 \pi p-(\pi / M), 2 \pi p+(\pi / M)], p=$ $0, \pm 1, \pm 2, \cdots$ and $\operatorname{Supp}\left(G_{l}\right)=\left[2 \pi q-\left(\pi / m_{l}\right), 2 \pi q+\left(\pi / m_{l}\right]\right.$, $q=0, \pm 1, \pm 2, \cdots$. Since the period of $H_{r_{l}+i}(\omega)$ is $2 \pi$, for each $v$, the period of $\bar{H}_{r_{l}+i}\left(\omega-\left(2 \pi v / m_{l}\right)\right)$ is $2 \pi M / m_{l}$. Therefore, we only need to focus on the region $-\left(\pi M / m_{l}\right) \leq \omega \leq$ $\left(\pi M / m_{l}\right)$, where

$$
p=0 \text { and }|q| \leq\left\lfloor\frac{M}{m_{l}}\right\rfloor
$$

where $\lfloor x\rfloor$ denotes the nearest integer less than or equal to $x$. From (10), we have

$$
\begin{aligned}
\operatorname{Supp} & \left(\bar{H}_{r_{l}+i}\left(\omega-\frac{2 \pi v}{m_{l}}\right)\right) \\
= & \operatorname{Supp}\left(\bar{H}_{r_{l}+i}^{+}\left(\omega-\frac{2 \pi v}{m_{l}}\right)\right) \\
& \bigcup \operatorname{Supp}\left(\bar{H}_{r_{l}+i}^{-}\left(\omega-\frac{2 \pi v}{m_{l}}\right)\right) \\
= & \left\{\omega \mid \frac{m_{l} \omega-2 \pi v}{M}-\omega_{r_{l}+i} \in\left[-\frac{\pi}{M}, \frac{\pi}{M}\right]\right\} \\
& \bigcup\left\{\omega \mid \frac{m_{l} \omega-2 \pi v}{M}+\omega_{r_{l}+i} \in\left[-\frac{\pi}{M}, \frac{\pi}{M}\right]\right\} \\
= & x_{v, r_{l}+i}^{+} \cup x_{v, r_{l}+i}^{-}
\end{aligned}
$$

where

$$
\begin{aligned}
x_{v, r_{l}+i}^{+} & =\left[\frac{\pi}{m_{l}}\left(2 v+r_{l}+i-\frac{1}{2}\right), \frac{\pi}{m_{l}}\left(2 v+r_{l}+i+\frac{3}{2}\right)\right] \\
& =\left[s_{v, r_{l}+i}^{+}, t_{v, r_{l}+i}^{+}\right] \\
x_{v, r_{l}+i}^{-} & =\left[\frac{\pi}{m_{l}}\left(2 v-\left(r_{l}+i\right)-\frac{3}{2}\right), \frac{\pi}{m_{l}}\left(2 v-\left(r_{l}+i\right)+\frac{1}{2}\right)\right] \\
& =\left[s_{v, r_{l}+i}^{-}, t_{v, r_{l}+i}^{-}\right] .
\end{aligned}
$$

Similarly from (11), we have

$$
\begin{aligned}
& \operatorname{Supp}\left(G_{l, i_{-} F B}(\omega)\right) \\
&= \operatorname{Supp}\left(G_{l, i}^{+}(\omega)\right) \bigcup \operatorname{Supp}\left(G_{l, i}^{-}(\omega)\right) \\
&=\left\{\omega \mid \omega-\eta_{l, i} \in\left[2 \pi q-\frac{\pi}{m_{l}}, 2 \pi q+\frac{\pi}{m_{l}}\right]\right. \\
&\left.q=0, \pm 1, \cdots,\left\lfloor\frac{M}{m_{l}}\right\rfloor\right\} \\
& \bigcup\left\{\omega \mid \omega+\eta_{l, i} \in\left\lfloor 2 \pi q-\frac{\pi}{m_{l}}, 2 \pi q+\frac{\pi}{m_{l}}\right]\right. \\
&\left.\left.q=0, \pm 1, \cdots, \mid \frac{M}{m_{l}}\right\rfloor\right\} \\
&=\left\{\bigcup_{q} y_{q, i}^{+}\right\} \cup\left\{\bigcup_{q} y_{q, i}^{-}\right\}
\end{aligned}
$$

where

$$
\begin{aligned}
y_{q, i}^{+} & =\left[\frac{\pi}{m_{l}}\left(2 m_{l} q+i-\frac{1}{2}\right), \frac{\pi}{m_{l}}\left(2 m_{l} q+i+\frac{3}{2}\right)\right] \\
& =\left[u_{q, i}^{+}, v_{q, i}^{+}\right] \\
y_{q, i}^{-} & =\left[\frac{\pi}{m_{l}}\left(2 m_{l} q-i-\frac{3}{2}\right), \frac{\pi}{m_{l}}\left(2 m_{l} q-i+\frac{1}{2}\right)\right] \\
& =\left[u_{q, i}^{-}, v_{q, i}^{-}\right] .
\end{aligned}
$$

It can be verified that the four intervals in (18) and (20) (for fixed $v$ and $q$ ) are equal to

$$
I=\frac{2 \pi}{m_{l}} .
$$

Support of $D_{1}^{(v)}(\omega)$ : Consider $D_{1}^{(v)}(\omega)=$ $(1 / 4) \sum_{i=0}^{m_{l}-1} e^{j\left(\theta_{r_{l}+i}+\xi_{l, i}\right)} \bar{H}_{r_{l}+i}^{+}\left(\omega-\left(2 \pi v / m_{l}\right)\right) G_{l, i}^{+}(\omega)$ in (15). The support of $\bar{H}_{r_{l}+i}+\left(\omega-\left(2 \pi v / m_{l}\right)\right) G_{l, i}^{+}(\omega)$ is $\operatorname{Supp}\left(x_{v, r_{l}+i}^{+} \cap y_{q, i}^{+}\right)$. To determine whether they overlap each other, we examine the difference between the starting locations of the two intervals. From (18) and (20), we get

$$
\begin{aligned}
s_{v, r_{l}+i}^{+}-u_{q, i}^{+} & =\frac{\pi}{m_{l}}\left(2 v+r_{l}+i-\frac{1}{2}\right)-\frac{\pi}{m_{l}}\left(2 m_{l} q+i-\frac{1}{2}\right) \\
& =\left(v+\frac{r_{l}}{2}-m_{l} q\right) I \\
& =\left(v+r_{l}^{\prime}-m_{l} q\right) I
\end{aligned}
$$

where $r_{l}^{\prime}=r_{l} / 2$ is an integer, because $r_{l}$ is even. From (22), we obtain

$$
\begin{cases}{\left[s_{v, r_{l}+i}^{+}, t_{v, r_{l}+i}^{+}\right]=\left[u_{q, i}^{+}, v_{q, i}^{+}\right],} & \text {if } v=m_{l} q-r_{l}^{\prime} \\ {\left[s_{v, r_{l}+i}^{+}, t_{v, r_{l}+i}^{+}\right] \cap\left[u_{q, i}^{+}, v_{q, i}^{+}\right]=\phi,} & \text { if } v \neq m_{l} q-r_{l}^{\prime}\end{cases}
$$




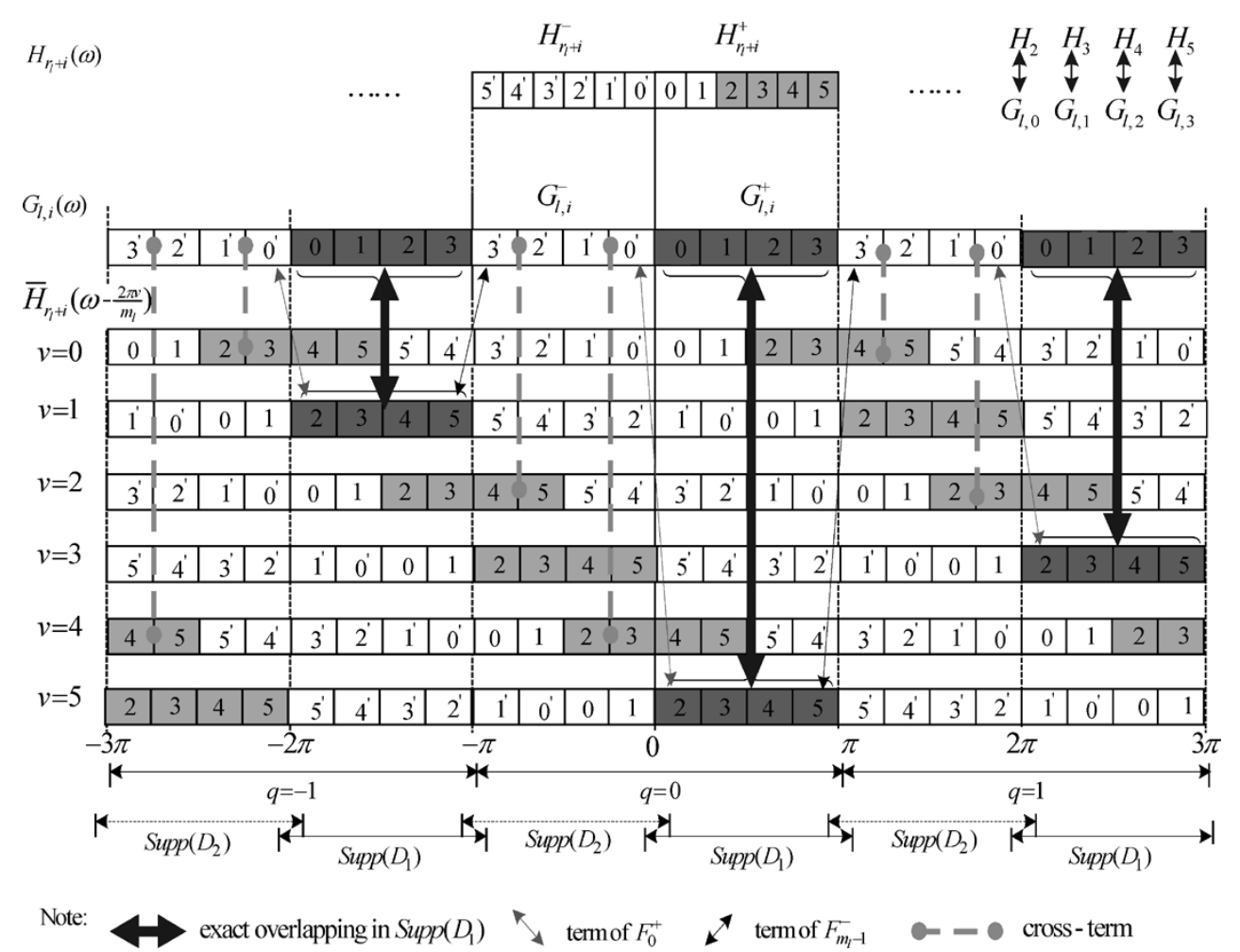

Fig. 5. Spectra of ideal filters $H_{r_{l}+i}(\omega), G_{l, i}(\omega)$ and $\bar{H}_{r_{l}+i}\left(\omega-\left(2 v \pi / m_{l}\right)\right)$ for the subband with sampling factor $4 / 6$ at the starting merging index of 2 .

If $v=m_{l} q-r_{l}^{\prime}$, the two intervals overlap exactly and it constitutes part of the desired passband of an ideal LTI analysis filter. Note, because $Y_{l}(\omega)$ has a period of $2 \pi M / m_{l}$, there are several copies of such response in one period of $Y_{l}(\omega)$, corresponding to each value of $q$ in the range $0 \leq q \leq\left\lfloor M / m_{l}\right\rfloor$ (the corresponding $v$ is $\left.v=m_{l} q-r_{l}^{\prime}\right)$. The support of one such copy is

$$
\begin{aligned}
& \operatorname{Supp}\left(\bar{H}_{r_{l}+i}^{+}\left(\omega-\frac{2 \pi v}{m_{l}}\right) G_{l, i}^{+}(\omega)\right) \\
& =\left[s_{v, r_{l}+i}^{+}, t_{v, r_{l}+i}^{+}\right] \\
& =\left[\frac{\pi}{m_{l}}\left(2 v+r_{l}+i-\frac{1}{2}\right), \frac{\pi}{m_{l}}\left(2 v+r_{l}+i+\frac{3}{2}\right)\right] .
\end{aligned}
$$

Therefore

$$
\begin{aligned}
& \operatorname{Supp}\left(D_{1}^{(v)}\right) \\
& \quad=\bigcup_{i=0,1, \cdots, m_{l}-1} \operatorname{Supp}\left(\bar{H}_{r_{l}+i}^{+}\left(\omega-\frac{2 \pi v}{m_{l}}\right) G_{l, i}^{+}(\omega)\right) \\
& =\left[\frac{\pi}{m_{l}}\left(2 v+r_{l}-\frac{1}{2}\right), \frac{\pi}{m_{l}}\left(2 v+r_{l}+m_{l}+\frac{1}{2}\right)\right] .
\end{aligned}
$$

The bandwidth of $D_{1}^{(v)}(\omega)$ is $\left(1+\left(1 / m_{l}\right)\right) \pi$. For ideal filters, the bandwidth is $\pi$. Note, $D_{1}^{(v)}(\omega)$ corresponds to the positive frequency part of the desired passband of the filter, and its conjugate is at $D_{4}^{(v)}(\omega)$. This is illustrated in Fig. 5, where the last four channels of a 6-channel uniform FB are merged by a 4-channel TMUX to yield a subband with a sampling factor of $4 / 6$. In this figure, each square box labeled by " $k$ " and " $k$ " $\left(k=0,1, \cdots, 5\right.$ and $\left.k^{\prime}=0^{\prime}, 1^{\prime}, \cdots, 5^{\prime}\right)$ in the row labeled
" $H_{r_{l}+i}(\omega)$ " corresponds to the ideal support of $H_{k}^{+}(\omega)$ and $H_{k}^{-}(\omega)$. Note, $H_{k}(\omega)$ represents all the analysis filters of the uniform CMFB, whereas $H_{r_{l}+i}(\omega)$ as shown in the figure denotes only those analysis filters to be merged having the index $r_{l}+i=k$, where $r_{l}$ is the starting index. For instance, suppose $r_{l}=2$ and $i=0,1,2,3$, then the filters to be merged will be $H_{k}(\omega)$ with $k=2,3,4,5$. Similar notations apply to the boxes in the rows labeled " $\bar{H}_{r_{l}+i}\left(\omega-\left(2 \pi v / m_{l}\right)\right)$ " with " $v=0$ " to " $v=5$." Similarly, each square box labeled by " $i$ " and " $i$ " $\left(i=0,1,2,3\right.$ and $\left.i^{\prime}=0^{\prime}, 1^{\prime}, 2^{\prime}, 3^{\prime}\right)$ in the row labeled " $G_{l, i}(\omega)$ " corresponds to the ideal supports of $G_{l, i}^{+}(\omega)$ and $G_{l, i}^{-}(\omega)$. The top-right corner of the figure indicates that, in the merging operation, there exists a one to one correspondence between $H_{2}(\omega) \sim H_{5}(\omega)$ and $G_{l, 0}(\omega) \sim G_{l, 3}(\omega)$. In Fig. 5, the gray colored boxes for $H_{r_{l}+i}(\omega), G_{l, i}(\omega)$ and $\bar{H}_{r_{l}+i}(\omega-$ $\left.\left(2 \pi v / m_{l}\right)\right)$ show the support to be merged. Here only those with superscript "+" are marked. From (8), $A_{v}(\omega)$ is obtained by multiplying $G_{l, i}(\omega)$ with $H_{r_{l}+i}\left(m_{l} \omega-2 \pi v / M\right)$ and sum over $i=0,1, \cdots, m_{l}-1$. For example, consider $A_{0}(\omega)$ [cf. (14)] in Fig. 5. The support of $G_{l, 0}^{+}(\omega)$ does not overlap exactly with the support of $\bar{H}_{r_{l}+0}^{+}\left(\omega-\left(2 \pi v / m_{l}\right)\right)=\bar{H}_{2}^{+}\left(\omega-\left(2 \pi v / m_{l}\right)\right)$ (the box " 0 " in row " $G_{l, i}(\omega)$ " does not overlap exactly with the position of box " 2 " in the row " $\bar{H}_{r_{l}+i}\left(\omega-\left(2 \pi v / m_{l}\right), v=0\right.$ "). Similarly, there is no exact overlapping for the remaining is for $v=0$. As a result, $A_{0}(\omega)$ will be close to zero. In order for $G_{l, i}^{+}(\omega)$ and $\bar{H}_{r_{l}+i}^{+}\left(\omega-\left(2 \pi v / m_{l}\right)\right)$ to overlap, the value $v$ should be such that their supports occupy the same position. In other words, box " $i$ " in " $G_{l, i}(\omega)$ " should be matched to box " $r_{l}+i$ " in $H_{r_{l}+i}\left(m_{l} \omega-2 \pi v / M\right)$. This occurs when "...,v=1 for $q=-1 ; v=5$ for $q=0 ; v=3$ for $q=1, \ldots$. " (As indicated by the thick vertical arrows in the figure.) These are the solutions to the congruent in (23), which gives rise to $D_{1}^{(v)}(\omega)$ 


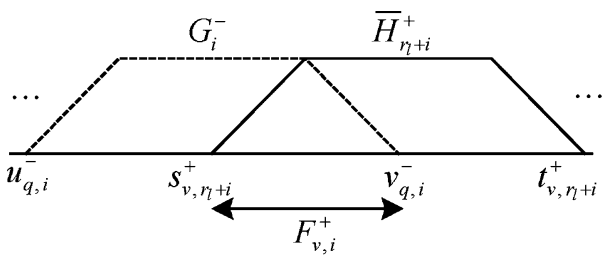

(a)

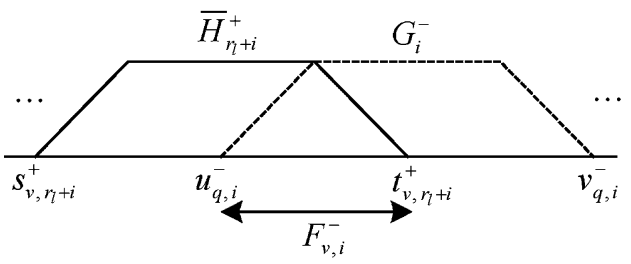

(b)

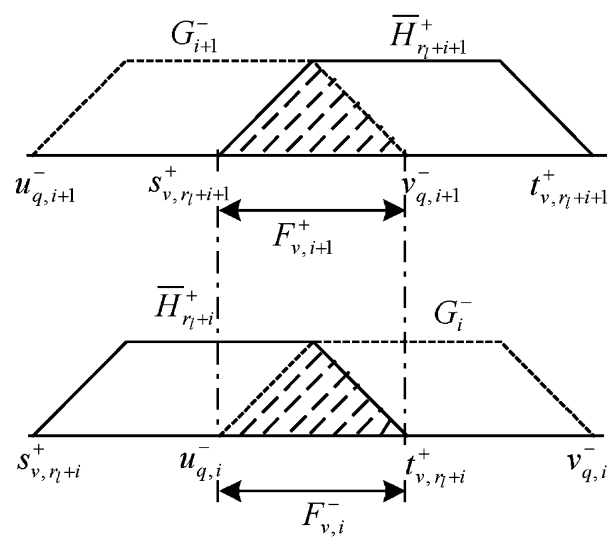

(c)

Fig. 6. Spectral supports of (a) $F_{v, i}^{+}$. (b) $F_{v, i}^{-}$. (c) Illustration of $\bar{H}_{r_{l}+i+1}^{+} G_{i+1}^{-} E_{F_{v, i+1}^{+}}=\bar{H}_{r_{l}+i}^{+} G_{i}^{-} E_{F_{v, i}}$.

with an ideal support of $\pi$ and a period of $2 \pi$. As aforementioned, it forms the positive frequency part of the desired response, while $D_{4}^{(v)}(\omega)$, which is the exact overlap of $G_{l, i}^{-}(\omega)$ and $\bar{H}_{r_{l}+i}^{-}\left(\omega-\left(2 \pi v / m_{l}\right)\right)$, is periodic with a period of $2 \pi$ and it occupies the spectrum from $[\pi, 2 \pi] . D_{1}^{(v)}(\omega)$ and $D_{4}^{(v)}(\omega)$ together constitute the complete desired spectrum with a period of $2 \pi$. For practical filters with finite support, transition bands of these frequency-shifted copies might overlap to yield undesirable or spurious responses and all of them are coming from $D_{2}^{(v)}(\omega)$. These are indicated by the dotted horizontal lines in Fig. 5. Next, we shall study their locations and suppression method.

Support of $D_{2}^{(v)}(\omega)$ : Consider $D_{2}^{(v)}(\omega)=$ $(1 / 4) \sum_{i=0}^{m_{l}-1} e^{j\left(\theta_{r_{l}+i}-\xi_{l, i}\right)} \bar{H}_{r_{l}+i}^{+}\left(\omega-\left(2 \pi v / m_{l}\right)\right) G_{l, i}^{-}(\omega)$. The support of $\bar{H}_{r_{l}+i}^{+}\left(\omega-\left(2 \pi v / m_{l}\right)\right) G_{l, i}^{-}(\omega)$ is $\operatorname{Supp}\left(x_{v, r_{l}+i}^{+} \cap y_{q, i}^{-}\right)$. Again, we examine the difference between the starting locations of the two intervals. From (18) and (20), we can see that

$$
\begin{aligned}
s_{v, r_{l}+i}^{+}-u_{q, i}^{-} & =\frac{\pi}{m_{l}}\left(2 v+r_{l}+i-\frac{1}{2}\right)-\frac{\pi}{m_{l}}\left(2 m_{l} q-i-\frac{3}{2}\right) \\
& =\left(v-m_{l} q+r_{l}^{\prime}+i+\frac{1}{2}\right) I .
\end{aligned}
$$

Because of the term 1/2 on the right-hand side (RHS) of (26), the sum inside the bracket cannot be zero. In other words, the spectrums cannot overlap exactly as in (22) for $D_{1}^{(v)}(\omega)$. However,

$$
\begin{array}{ll}
\text { if } & v-m_{l} q+r_{l}^{\prime}+i=0 \\
\text { or } & v-m_{l} q+r_{l}^{\prime}+i=-1
\end{array}
$$

then the transition bands of $\bar{H}_{r_{l}+i}^{+}\left(\omega-\left(2 \pi v / m_{l}\right)\right)$ and $G_{l, i}^{-}(\omega)$ will overlap with $s_{v, r_{l}+i}^{+}-u_{q, i}^{-}= \pm I / 2$ for (27) and (28), respectively. The two cases corresponding to (27) and (28) are illustrated in Fig. 6(a) and Fig. 6(b), respectively. From Fig. 6(a), we get

$$
\begin{aligned}
& {\left[s_{v, r_{l}+i}^{+}, t_{v, r_{l}+i}^{+}\right] \cap\left[u_{q, i}^{-}, v_{q, i}^{-}\right]} \\
& \quad=\left[s_{v, r_{l}+i}^{+}, v_{q, i}^{-}\right] \\
& \quad=F_{v, i}^{+} \\
& \quad=\left[\frac{\pi}{m_{l}}\left(2 v+r_{l}+i-\frac{1}{2}\right), \frac{\pi}{m_{l}}\left(2 m_{l} q-i+\frac{1}{2}\right)\right] .
\end{aligned}
$$

Similar from Fig. 6(b), which satisfying (28), we have

$$
\begin{aligned}
& {\left[s_{v, r_{l}+i}^{+}, t_{v, r_{l}+i}^{+}\right] \cap\left[u_{q, i}^{-}, v_{q, i}^{-}\right]} \\
& \quad=\left[u_{q, i}^{-}, t_{r_{l}+i}^{+}\right] \\
& \quad=F_{v, i}^{-} \\
& \quad=\left[\frac{\pi}{m_{l}}\left(2 m_{l} q-i-\frac{3}{2}\right), \frac{\pi}{m_{l}}\left(2 v+r_{l}+i+\frac{3}{2}\right)\right]
\end{aligned}
$$

Comparing (29) and (30), one gets $F_{v, i+1}^{+}=F_{v, i}^{-}$. Denote $E_{F}$ as the indicator function of the set $F$, where $E_{F}(\omega)=\left\{\begin{array}{ll}1, & \text { if } \omega \in F \\ 0, & \text { if } \omega \notin F\end{array}\right.$. Hence

$$
\begin{aligned}
D_{2}^{(v)}(\omega)= & \frac{1}{4} \sum_{i=0}^{m_{l}-1} e^{j\left(\theta_{r_{l}+i}-\xi_{l, i}\right)} \bar{H}_{r_{l}+i}^{+}\left(\omega-\frac{2 \pi v}{m_{l}}\right) G_{l, i}^{-}(\omega) \\
= & \frac{1}{4} e^{j\left(\theta_{r_{l}}-\xi_{l, 0}\right)} \bar{H}_{r_{l}}^{+}\left(\omega-\frac{2 \pi v}{m_{l}}\right) G_{l, 0}^{-}(\omega) E_{F_{v, 0}^{+}} \\
& +\frac{1}{4} \sum_{i=0}^{m_{l}-2}\left(e^{j\left(\theta_{r_{l}+i+1}-\xi_{l, i+1}\right)} \bar{H}_{r_{l}+i+1}^{+}\left(\omega-\frac{2 \pi v}{m_{l}}\right)\right. \\
& \times G_{l, i+1}^{-}(\omega) E_{F_{v, i+1}^{+}}+e^{j\left(\theta_{r_{l}+i-\xi_{l, i}}\right)} \\
& +\frac{1}{4} e^{j\left(\theta_{r_{l}+m_{l}-1}-\xi_{l, m_{l}-1}\right)} \\
& \left.\times \bar{H}_{r_{l}+i}^{+}\left(\omega-\frac{2 \pi v}{m_{l}}\right) G_{l, i}^{-}(\omega) E_{F_{v, i}^{-}}\right) \\
& \left(\omega-\frac{2 \pi v}{m_{l}}\right) G_{l, m_{l}-1}^{-} E_{F_{v, m_{l}-1}^{-}} \cdot(3)
\end{aligned}
$$


The term $\quad \sum_{i=0}^{m_{l}-2}\left(e^{j\left(\theta_{r_{l}+i+1}-\xi_{l, i+1}\right)} \bar{H}_{r_{l}+i+1}^{+}(\omega \quad\right.$ $\left.\left(2 \pi v / m_{l}\right)\right) G_{l, i+1}^{-}(\omega) E_{F_{v, i+1}^{+}}+e^{j\left(\theta_{r_{l}+i}-\xi_{l, i}\right)} \bar{H}_{r_{l}+i}^{+}(\omega-$ $\left.\left.\left(2 \pi v / m_{l}\right)\right) G_{l, i}^{-}(\omega) E_{F_{v, i}^{-}}\right)$, which is called the cross-term, is the source of the spurious response.

Matching Condition: To suppress the spurious response, we propose the following matching condition:

$$
\begin{aligned}
\frac{N_{M}}{M} & =\frac{N_{m_{l}}}{m_{l}} \\
\bar{H}(\omega) & =G_{l}, \text { or equivalently } \\
H\left(\frac{m_{l}}{\omega_{M}}\right) & =G_{l}(\omega), \quad \text { for } \omega \in\left[\frac{-\pi}{m_{l}}, \frac{\pi}{m_{l}}\right] .
\end{aligned}
$$

From (12) and (13), we have

$$
\begin{aligned}
\bar{H}_{r_{l}+i}^{+} & \left(\omega-\frac{2 \pi v}{m_{l}}\right) G_{l, i}^{-}(\omega) \\
= & \bar{H}\left(\omega-\frac{2 \pi v}{m_{l}}-\frac{M}{m_{l}} \omega_{r_{l}+i}\right) G_{l}\left(\omega+\eta_{l, i}\right) \\
= & \bar{H}\left(\omega-\left(\frac{2 \pi v}{m_{l}}+\frac{\pi\left(2\left(r_{l}+i\right)+1\right)}{2 m_{l}}\right)\right) \\
& \times G_{l}\left(\omega-\left(2 \pi q-\frac{\pi(2 i+1)}{2 m_{l}}\right)\right) .
\end{aligned}
$$

Replacing $i$ in (34) by $i+1$ yields

$$
\begin{aligned}
\bar{H}_{r_{l}+i+1}^{+} & \left(\omega-\frac{2 \pi v}{m_{l}}\right) G_{l, i+1}^{-}(\omega) \\
= & \bar{H}\left(\omega-\frac{2 \pi v}{m_{l}}-\frac{M}{m_{l}} \omega_{r_{l}+i+1}\right) G_{l}\left(\omega+\eta_{l, i+1}\right) \\
= & \bar{H}\left(\omega-\left(\frac{2 \pi v}{m_{l}}+\frac{\pi\left(2\left(r_{l}+i\right)+3\right)}{2 m_{l}}\right)\right) \\
& \times G_{l}\left(\omega-\left(2 \pi q-\frac{\pi(2 i+3)}{2 m_{l}}\right)\right) .
\end{aligned}
$$

From (28), (35) becomes

$$
\begin{gathered}
\bar{H}_{r_{l}+i+1}^{+}\left(\omega-\frac{2 \pi v}{m_{l}}\right) G_{l, i+1}^{-}(\omega) \\
=G_{l}\left(\omega-\left(\frac{2 \pi v}{m_{l}}+\frac{\pi\left(2\left(r_{l}+i\right)+1\right)}{2 m_{l}}\right)\right) \\
\quad \times \bar{H}\left(\omega-\left(2 \pi q-\frac{\pi(2 i+1)}{2 m_{l}}\right)\right) .
\end{gathered}
$$

It can be seen that if (33) is satisfied, $\bar{H}($.$) and G_{l}($.$) in$ (34) will be equal to $G_{l}($.$) and \bar{H}($.$) in (36), respec-$ tively. So, we get $\bar{H}_{r_{l}+i}^{+}\left(\omega-\left(2 \pi v / m_{l}\right)\right) G_{l, i}^{-}(\omega) E_{F_{v, i}^{-}}=$ $\bar{H}_{r_{l}+i+1}^{+}\left(\omega-\left(2 \pi v / m_{l}\right)\right) G_{l, i+1}^{-}(\omega) E_{F_{v, i+1}^{+}}, \quad$ as illustrated in Fig. 6(c). The cross-term, therefore, reduces to $\sum_{i=0}^{m_{l}-2}\left(e^{j\left(\theta_{r_{l}+i+1}-\xi_{l, i+1}\right)}+e^{j\left(\theta_{r_{l}+i}-\xi_{l, i}\right)}\right) \bar{H}_{r_{l}+i}^{+}(\omega-$ $\left.\left(2 \pi v / m_{l}\right)\right) G_{l, i}^{-}(\omega) E_{F_{v, i}^{-}}$. Further, if (32) is satisfied, it can be shown that $e^{j\left(\theta_{r_{l}+i+1}-\xi_{l, i+1}\right)}+e^{j\left(\theta_{r_{l}+i}-\xi_{l, i}\right)}=0$. As a result, if the matching condition in (32) and (33) is satisfied, the cross-term is zero and the spurious response is suppressed. The support of $D_{2}^{(v)}(\omega)$ now reduces to

$$
\operatorname{Supp}\left(D_{2}^{(v)}\right)= \begin{cases}F_{v, 0}^{+} \cup F_{v, m_{l}-1}^{-}, & \text {for } v=m_{l} q-r_{l}^{\prime} \\ 0, & \text { for } v \neq m_{l} q-r_{l}^{\prime}\end{cases}
$$

where $F_{v, 0}^{+}=\left[\left(\pi / m_{l}\right)\left(2 v+r_{l}-(1 / 2)\right),\left(\pi / m_{l}\right)\left(2 m_{l} q+\right.\right.$ $(1 / 2))]$ and $F_{v, m_{l}-1}^{-}=\left[\left(\pi / m_{l}\right)\left(2 m_{l} q+m_{l}-\right.\right.$ $\left.(1 / 2)),\left(\pi / m_{l}\right)\left(2 v+r_{l}+m_{l}+(1 / 2)\right)\right]$. It suggests that when $v=m_{l} q-r_{l}^{\prime}, \operatorname{Supp}\left(D_{2}^{(v)}\right)$ belongs to $\operatorname{Supp}\left(D_{1}^{(v)}\right)$. Finally, we have

$$
\begin{aligned}
& D_{2}^{(v)}(\omega)=\frac{1}{4} e^{j\left(\theta_{r_{l}}-\xi_{l, 0}\right)} \bar{H}_{r_{l}}^{+}\left(\omega-\frac{2 \pi v}{m_{l}}\right) G_{l, 0}^{-}(\omega) E_{F_{v, 0}^{+}} \\
& +\frac{1}{4} e^{j\left(\theta_{r_{l}+m_{l}-1}-\xi_{l, m_{l}-1}\right)} \bar{H}_{r_{l}+m_{l}-1}^{+}\left(\omega-\frac{2 \pi v}{m_{l}}\right) G_{l, m_{l}-1}^{-} E_{F_{v, m_{l}-1}^{-}}
\end{aligned}
$$

The origin of the spurious response is illustrated using the previous example as shown in Fig. 5. In the region labeled $\operatorname{Supp}\left(D_{2}\right)$, the support of $G_{l, i}^{-}(\omega)$ and $\bar{H}_{r_{l}+i}^{+}\left(\omega-\left(2 \pi v / m_{l}\right)\right)$ cannot overlap exactly. However, the transition band of adjacent copies, such as $\left\{G_{l, 3}^{-}(\omega), \bar{H}_{5}^{+}\left(\omega-\left(2 \pi v / m_{l}\right)\right)\right\}$ and $\left\{G_{l, 2}^{-}(\omega), \bar{H}_{4}^{+}\left(\omega-\left(2 \pi v / m_{l}\right)\right)\right\}$ for $v=0$ and $q=1$, can overlap. These two spurious responses occupy the same location and if the matching condition is satisfied, then they will cancel each other. Similar spurious response pairs are shown in thick dotted line. Note, the overlapping of $G_{l, i}^{+}(\omega)$ and $\bar{H}_{r_{l}+i}^{-}\left(\omega-\left(2 \pi v / m_{l}\right)\right)$ are not shown here and they belong to $\operatorname{Supp}\left(D_{3}\right)$, the conjugate of $\operatorname{Supp}\left(D_{2}\right)$. The terms $F_{v, 0}^{+}$and $F_{v, m_{l}-1}^{-}$appear at the intersection of $\operatorname{Supp}\left(D_{1}\right)$ and $\operatorname{Supp}\left(D_{2}\right)$, and they constitute the two trail ends of the desired passband.

Note, although the matching conditions for both the coprime and noncoprime cases are almost identical, the behavior of the analysis filters are quite different. In the coprime case [5], the analysis filters are LTI and it can be written as the direct form in Fig. 2. For the noncoprime case, the filter is LPTV and the aliasing is suppressed to a level equal to the stopband attenuation of the modulated filters, except at the intersections of their transition bands. If the matching condition is satisfied, this major source of aliasing will also be suppressed. This is illustrated in Fig. 4, where the bumps in the bifrequency plane, Fig. 4(e), are suppressed to the same level of the other unwanted components when the matching condition is satisfied. For clarity, its projection is also plotted in Fig. 4(f). Since the FB is paraunitary, suppressing the undesirable and stopband components means that the passband ripples will be suppressed at the same time. We now describe the design procedure to enforce the matching condition.

\section{DESIGN PROCEDURE AND EXAMPLES}

Suppose that the passband and stopband cutoff frequencies of $H(\omega)$ are $\pi /(2 M)$ and $\left(1+2 \rho_{M}\right) \pi /(2 M)$, where $\rho_{M}$ is the roll-off factor and $0<\rho_{M}<1$. They are mapped, respectively, 


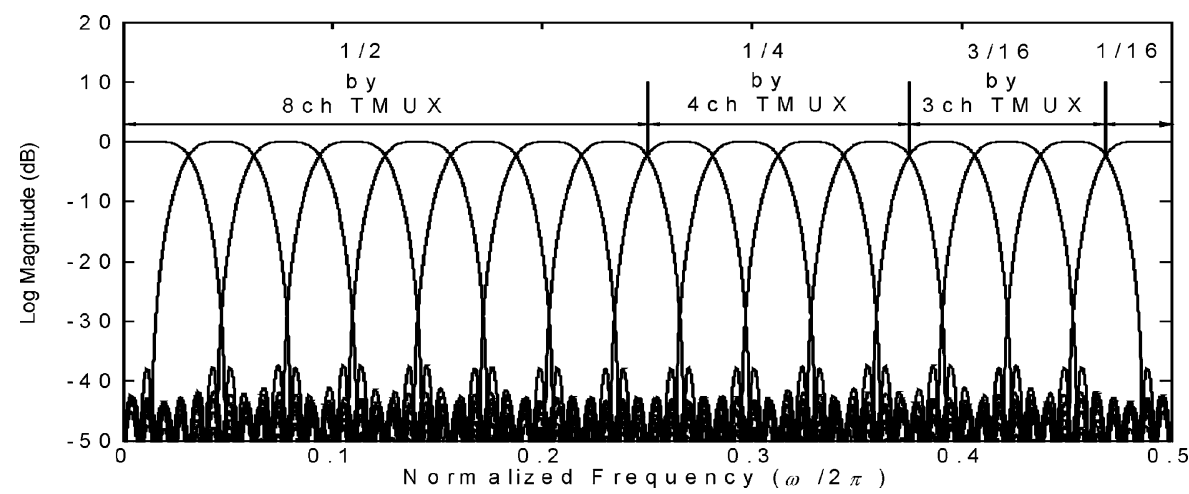

Fig. 7. Design example 1-multiband RN-CMFB. Frequency responses of the original 16-channel CMFB. (The recombination 8-, 4-, and 3-channel CMFBs are plotted in Figs. 8(e), 9(e), and 8(g), respectively).

to $\pi /\left(2 m_{l}\right)$ and $\left(1+2 \rho_{M}\right) \pi /\left(2 m_{l}\right)$, when $H(\omega)$ is downsampled and then upsampled to $H\left(M \omega / m_{l}\right)$. Similarly, the passband and stopband cutoff frequencies of $G_{l}(\omega)$ are $\pi /\left(2 m_{l}\right)$ and $\left(1+2 \rho_{m_{l}}\right) \pi /\left(2 m_{l}\right)$, where $0<\rho_{m_{l}}<1$. In order to satisfy (33), i.e., $H\left(m_{l} \omega / M\right)=G_{l}(\omega)$, the two roll-off factors should be identical: $\rho_{m_{l}}=\rho_{M}$. Therefore, all the prototype filters in a RNFB have similar shapes. In our analysis, we have assumed that $\rho=\rho_{m_{l}}=\rho_{M}$ lies between 0 and 0.5. In what follows, we will summarize the design procedure along with three examples so as to illustrate the design method and principle of the adaptive RN-CMFB. In addition, the computational complexity of the proposed method will be briefly analyzed.

\section{A. Design Procedure}

Given the sampling factors $\left\{m_{l} / M\right\}, l=0,1, \cdots, L-1$ with $\sum_{l=0}^{L-1} m_{l}=M$.

1) Design an $M$-channel uniform CMFB with prototype filter $H\left(e^{j \omega}\right)$, filter length $N_{M}=2 m M$, and cutoff frequency $\omega_{s}=(1+2 \rho) \pi /(2 M)$ using (4).

2) Set $N_{m_{l}}=m_{l} N_{M} / M=2 m m_{l}$ (matching condition (32)).

3) Design the $m_{l}$-channel uniform recombination CMFB with length $N_{m_{l}}$, cutoff frequency $\omega_{s}=(1+2 \rho) \pi /\left(2 m_{l}\right)$, and prototype filter $G_{l}\left(e^{j \omega}\right)$ using (4). The weighting in the passband and stopband should be identical to the $M$-channel CMFB. This ensures that the matching condition in (33) is approximately satisfied. ${ }^{3}$

4) Obtain the corresponding CMFB-based PR TMUX as $G_{l, i}(\omega)=e^{-j \omega} G_{l, i_{-} F B}(\omega)$.

5) If all starting indexes $r_{l}$ are even, the subbands of the $M$-channel CMFB can be merged directly by using the synthesis filters of the $m_{l}$-channel PR TMUX.

6) If any $r_{l}$ is odd, the sequence $(-1)^{n}$ should be multiplied to the corresponding channels before merging the subbands.

7) Compensate the delay caused by the inserted TMUX in the other branches of the $M$-channel uniform FB.

\footnotetext{
${ }^{3}$ Because the relative transition bandwidth of $G_{l}\left(e^{j \omega}\right)$ and $H\left(e^{j \omega}\right)$ are similar and the same least square objective function is used in the optimization, $G_{l}\left(e^{j \omega}\right)$ so obtained will be approximately equal to $H\left(e^{j\left(m_{l} / M\right) \omega}\right)$.
}

We now present some examples to illustrate the proposed design method and different aspects of the proposed RN-CMFBs.

\section{B. Design Examples}

The optimization problem (4) in designing the PR CMFBs are solved using the DNCONF subroutine of the IMSL library, which is based on the sequential quadratic programming (SQP) for solving constrained optimization problem. The prototype filters are designed independently according to the procedure described above and the PR violation is of the order of $10^{-15}$. The optimization program converges, on the average, in about 150 iterations.

Example 1: Multiband PR RN-CMFB: In this example, a PR nonuniform FB with sampling factors $(1 / 2,1 / 4,3 / 16,1 / 16)$ is considered as a comparison to the pseudo-PR CMFB in [16]. A 16-channel uniform PR CMFB is first designed. Then, the first eight channels, the next four channels and the reminding three channels of this FB are, respectively, merged by an 8-, 4-, and 3-channel PR CMFB-based TMUXs to realize the desired sampling factors of $(8 / 16,4 / 16,3 / 16,1 / 16)=(1 / 2,1 / 4,3 / 16,1 / 16)$. The PR CMFB and TMUXs have to satisfy the above matching condition. Figs. 7, 8(e), 9(e), and 8(g) show the frequency responses of the original 16-channel CMFB and the recombination 8-, 4-, and 3-channel CMFBs, respectively. The filter lengths and the implementation complexity are summarized in Table I. Since the rate-1/2 (8/16) and the rate-1/4 (4/16) channels do not satisfy the coprime condition, the analysis filters are LPTV and there is no equivalent LTI representation like Fig. 2. The stopband attenuation of the LPTV filter is around $40 \mathrm{~dB}$, which is worse than that of the nonuniform FB in [16] (which is around $50 \mathrm{~dB}$ ). The latter has a PR violation of $10^{-3}$. The system delays of the recombination structure and the direct structure are, respectively, 383 and 169 samples. We can see that the pseudo-PR CMFB has a lower system delay, but higher complexity than the PR recombination system. This difference in system delay can be reduced by using low-delay biorthogonal CMFBs, instead of their orthogonal counterparts employed here. The details on the design of PR biorthogonal RN-CMFBs can be found in [6, 27]. Finally, we remark that the required sampling factors can also be realized by combining the RNFB with traditional tree-structured FBs. 


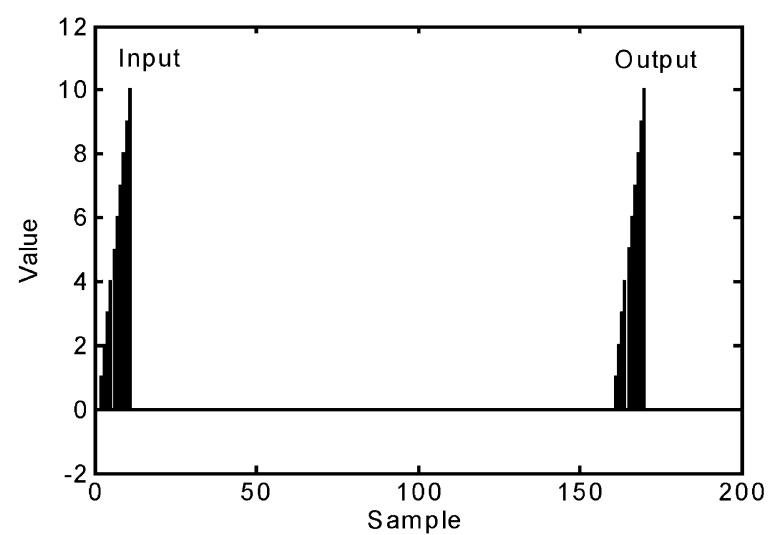

(a)
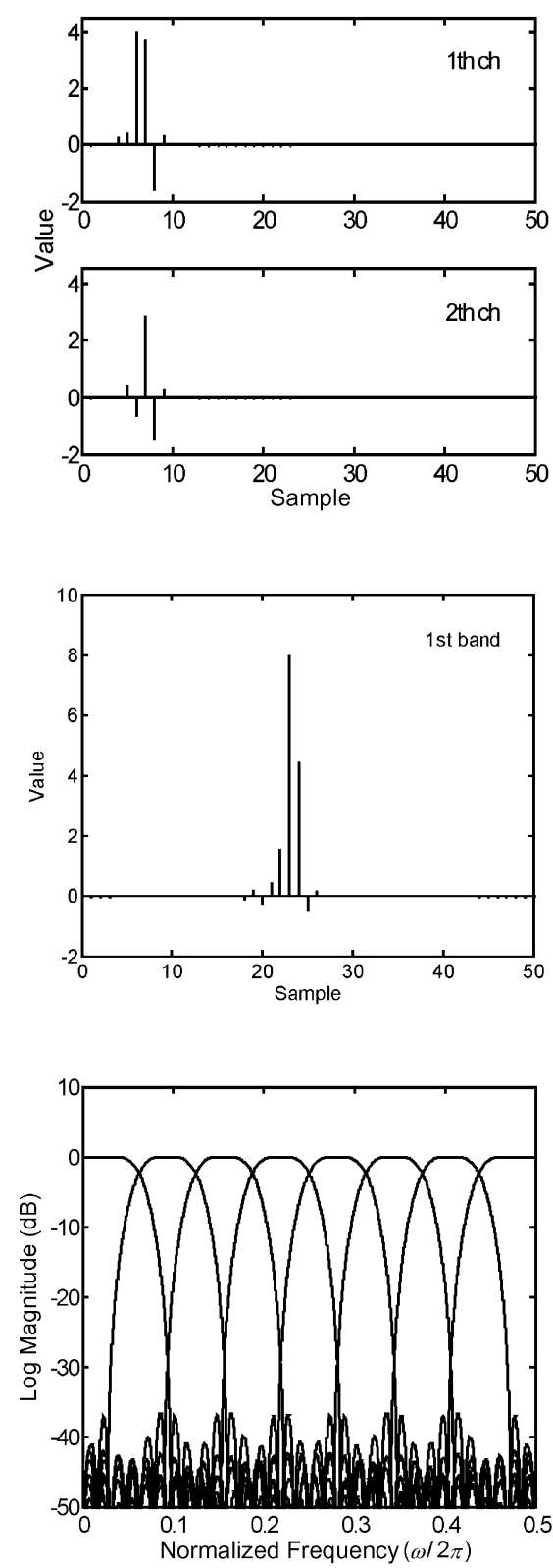

(e)

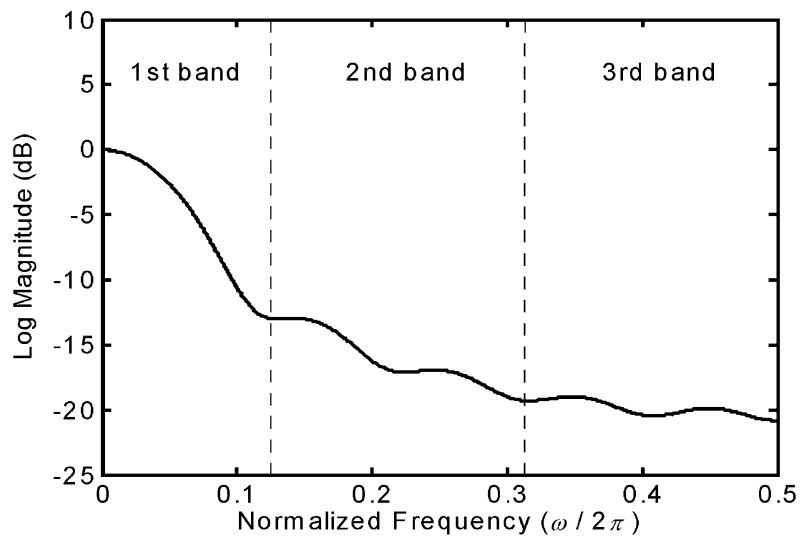

(b)

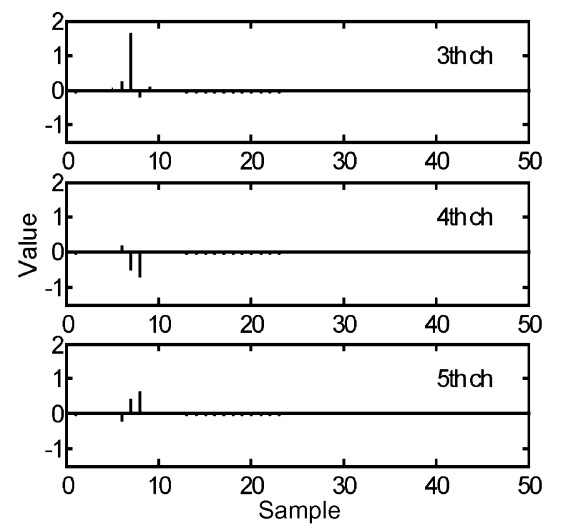

(c)

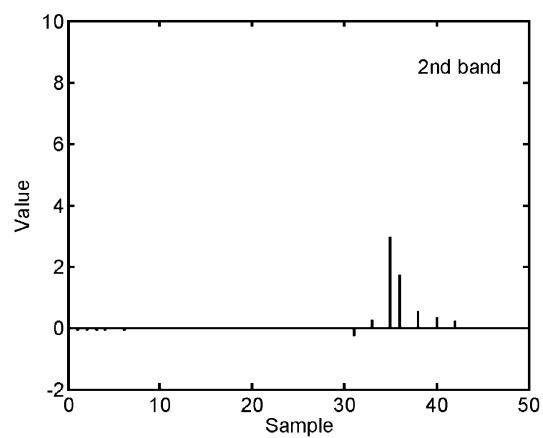

(d)

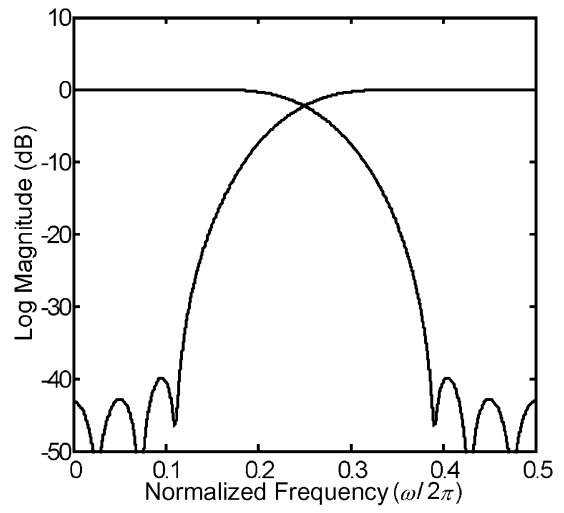

(f)
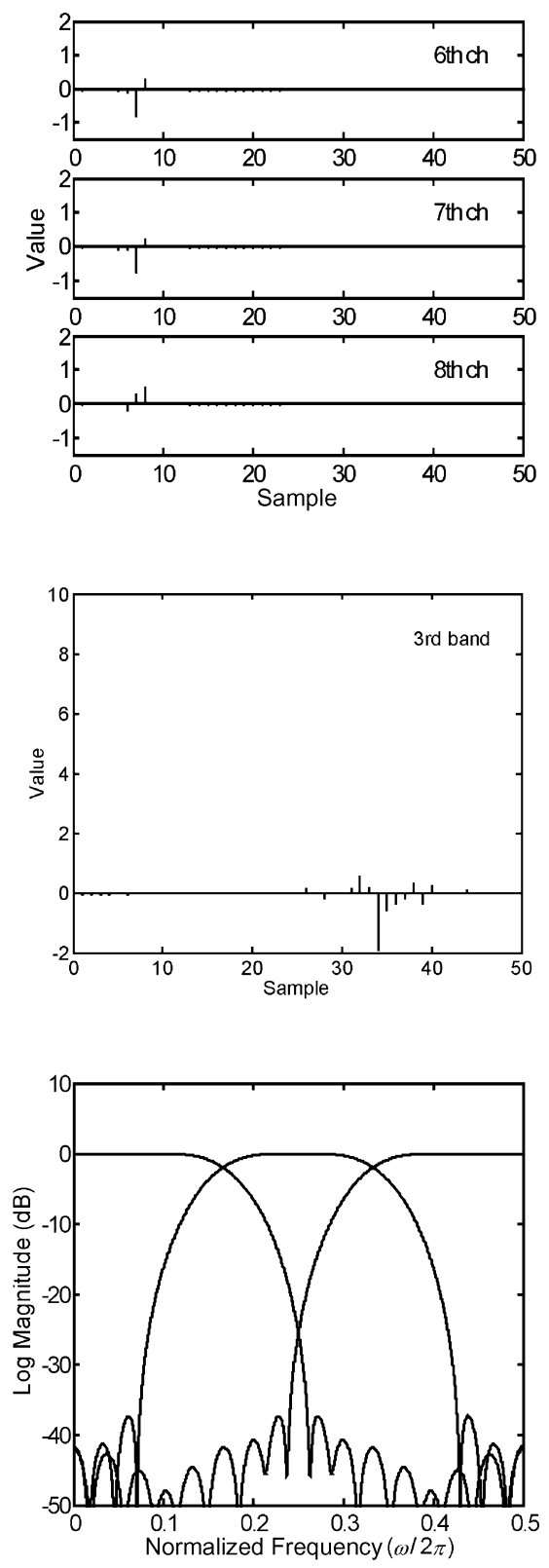

(g)

Fig. 8. Design results of example 2-RN-CMFB with different time-frequency resolution. (a) Input signal. (b) Its frequency spectrum. (c) Outputs of the 8-channel uniform FB. (d) Merged outputs the first band, second band, and third bands, frequency responses of the (e) 8-channel; (f) 2-channel; and (g) 3-channel CMFBs. (Sampling factors $=(2 / 8,3 / 8,3 / 8))$. 


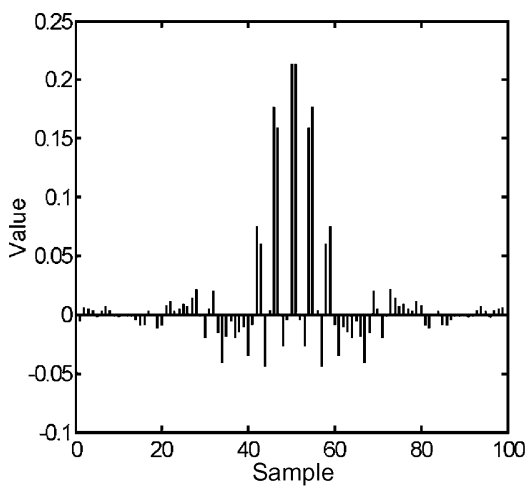

(a)

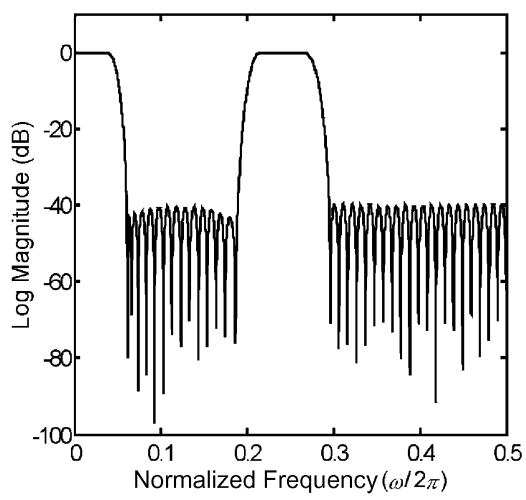

(b)

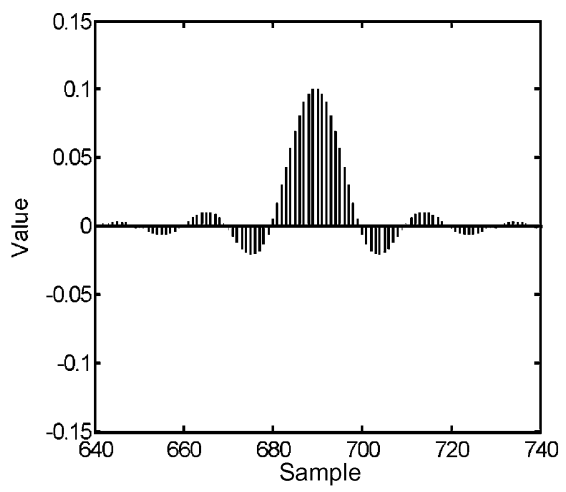

(c)

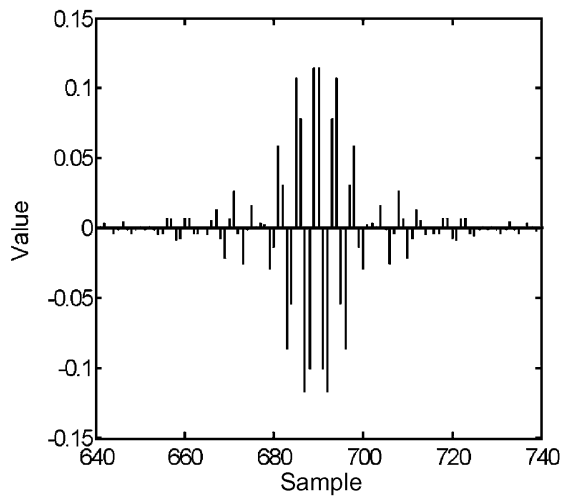

(d)

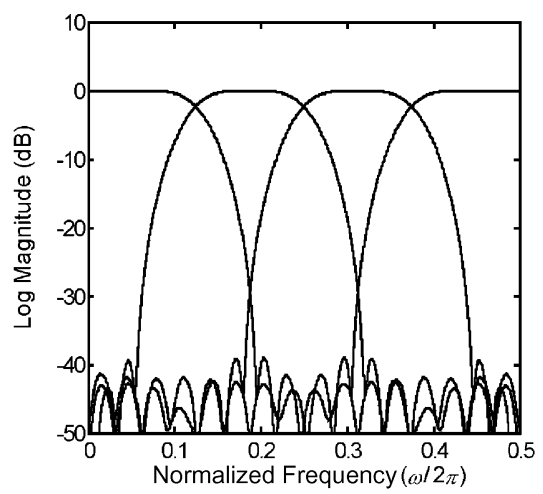

(e)

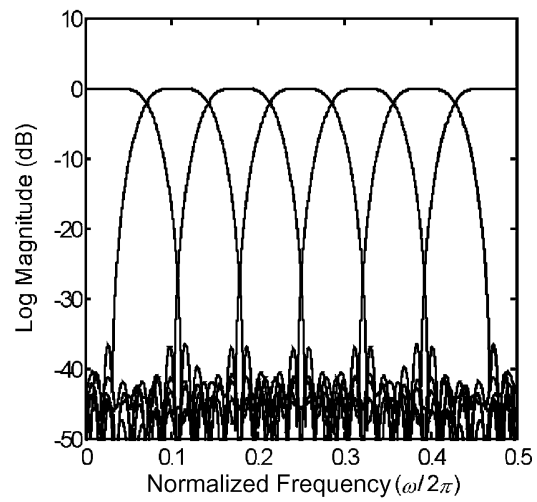

(f)

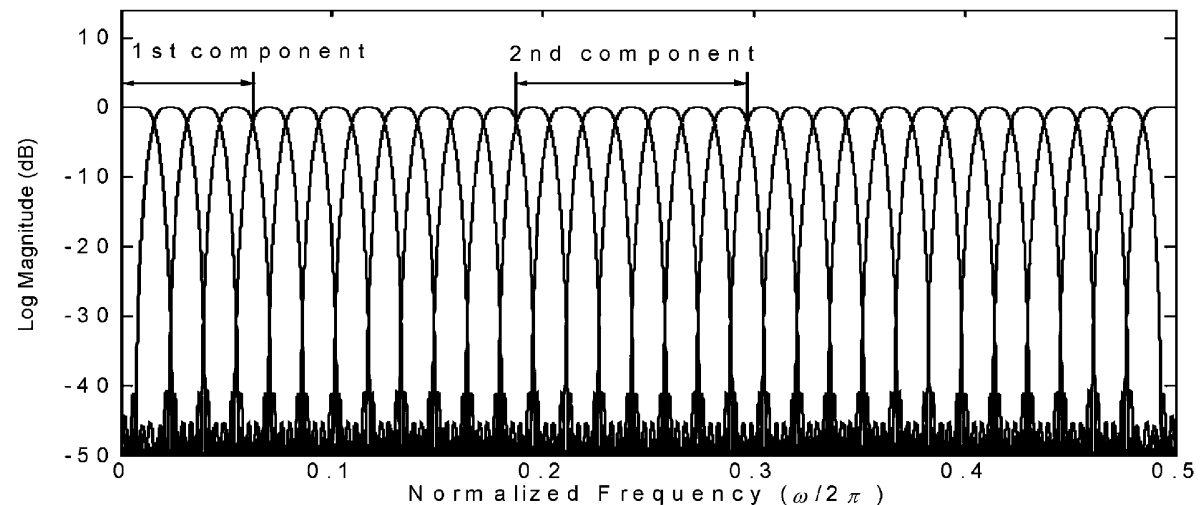

(g)

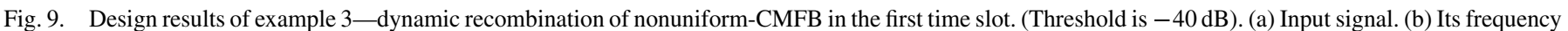

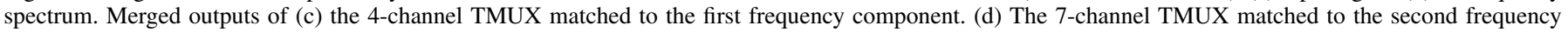
component. Frequency responses of the (e) 4-channel; (f) 7-channel; and (g) 32-channel CMFBs.

For example, two 2-channel uniform FBs can be cascaded to form a tree structure with sampling factors $(1 / 2,1 / 4,1 / 4)$. A RN-CMFB with sampling factors $(3 / 4,1 / 4)$ can be cascaded to the third branch of the tree structure to realize the desired sampling factors. ${ }^{4}$ This suggests that in a number of cases the RN-CMFB can be combined with traditional tree-structured FBs to realize more flexible structures. Table I summarizes the arithmetic complexity of the proposed PR RN-CMFB and the pseudo-PR CMFB in [16]. The detailed analysis will be deferred to the next subsection. The complexity is measured by the number of the required multiplications per unit-time (MPU) and additions per unit time (APU). Notice that since the filter

${ }^{4}$ The system delay of this FB is usually higher than the noncoprime RN-CMFBs described above. Due to space limitation, the details are omitted. lengths $N_{0}$ to $N_{3}$ used in [16] were not multiples of the upand down-sampling factors they were rounded to the nearest value as shown in Table I for the sake of presentation. Columns 3 and 4 give, respectively, the required MPU and APU for the direct structure and the proposed PR recombination structure. In the direct structure, the complexity for both the polyphase and direct-form implementation are shown. It can be seen that even in the more efficient polyphase implementation, the computational complexity of the direct structure (MPU $=264$ and $\mathrm{APU}=263$ ) is still considerably higher than that of the recombination structures $(\mathrm{MPU}=23$ and $\mathrm{APU}=23$ ). The low complexity of the RN-CMFB is mainly due to the interpolated filter structure adopted in the RN-CMFB, where the polyphase components can be moved to the lowest rate part 
TABLE I

COMPARISON OF DifFERENT STRUCTURES IN TERMS OF IMPLEMENTATION COMPLEXITY. (THE COST OF MODULATION IN THE CMFB IS Not COUNTED FOR SIMPLICITY)

\begin{tabular}{c|c|c|c}
\hline $\begin{array}{c}\text { Sampling factors of } \\
\text { Nonuniform FB }\end{array}$ & $\begin{array}{c}\text { Filter Length and } \\
\text { Complexity for Analysis } \\
\text { Bank }\end{array}$ & $\begin{array}{c}\text { Direct Structure } \\
\text { Pseudo-PR [16] }\end{array}$ & $\begin{array}{c}\text { PR Recombination } \\
\text { Structure }\end{array}$ \\
\hline \multirow{2}{*}{ Example 1: } & Filter Length & $\begin{array}{c}N_{0}=N_{1}=N_{3}=170 \\
(160) \\
N_{2}=508(480)\end{array}$ & $\begin{array}{c}N_{M}=192, N_{m_{0}}=96 \\
N_{m_{1}}=48, N_{m_{2}}=36\end{array}$ \\
\cline { 2 - 4 }$\left(\frac{1}{2}, \frac{1}{4}, \frac{3}{16}, \frac{1}{16}\right)$ & MPU & $264\{* 2034\}$ & 23 \\
\cline { 2 - 4 } & APU & $263\{* 2028\}$ & 23 \\
\hline
\end{tabular}

$\left\{*_{-}\right\}$Direct implementation without using polyphase decomposition.

of the system. Further, the CMFB in the proposed RN-CMFB can be implemented as the lossless lattice structure in [12], [13], which is more robust to coefficient round-off error than the direct structure.

Example 2: RN-CMFB With Different Time Frequency Resolution: In this example, we show how the recombination approach can be used to improve the time-resolution of a uniform CMFB by merging its subband outputs. Suppose that the input is a ramp signal as shown in Fig. 8(a) and its DTFT or frequency spectrum is shown in Fig. 8(b). This signal is passed through an 8-channel uniform CMFB with length 80 and roll-off factor $\rho_{M}=0.5$. The outputs are shown in Fig. 8(c). A RNFB with sampling factors $(2 / 8,3 / 8,3 / 8)$ is then derived from this uniform CMFB by merging its first two channels by a 2-channel TMUX and the remaining six channels by two 3-channel TMUXs. To suppress the undesirable spurious response mentioned earlier, the lengths of the 2- and 3-channel orthogonal uniform TMUXs are chosen to be 20 and 30 taps, respectively, and their roll-off are chosen to be $\rho=\rho_{m_{l}}=\rho_{M}=0.5$ in order to satisfy approximately the matching condition in (32) and (33). The uniform and recombination CMFBs designed are shown in Fig. 8(e), (f), and (g). As shown in Fig. 8(a), the input signal is completely reconstructed with a delay of about 160 samples. Fig. 8(d) shows the merged outputs of the first, second, and third outputs of the RN-CMFB. Comparing the first figure in Fig. 8(d) and the first two figures in Fig. 8(c), which show the outputs of the first and second channels in the 8-channel uniform FB, it can be seen that the time resolution of the merged subband is improved at the expense of reduced frequency resolution. Within the RN-CMFB, the low frequency band (rate-1/4) has a slightly better frequency resolution than the other two bands, which have a slightly better time resolution (rate-3/8).

Example 3: Dynamic Recombination of RN-CMFB: In the RNFBs, the PR condition is structurally imposed, and the original and the recombination FBs can be designed separately. Therefore, dynamical merging of consecutive subbands of the $M$-channel uniform FB by predesigned TMUXs is possible. By so doing, adjustable or time-varying time-frequency resolution (partitioning) can be achieved. To this end, we need some kinds of measures to decide which frequency bands should be merged or partially reconstructed. For simplicity and illustrative purposes, we shall merge consecutive subbands if their variances are larger than a certain threshold. In other words, we assume that the individual signals are well separated in the frequency domain. The dynamic recombination algorithm is outlined as follows:

1) Decompose the input signal by an $M$-channel uniform CMFB.

2) Calculate the variance $\sigma_{k}^{2}(k=0,1, \cdots, M-1)$ of each subband in non-overlapping interval of duration $T_{D}=100$, which denotes a time slot or snapshot of the signal.

3) Label each subband with 0 or 1 by comparing its variance $\left(\sigma_{k}^{2}\right)$ with a threshold $T$.

4) Consecutive subbands with label 1 are then merged together, and it gives the number of channel $m_{l}$ of the recombination TMUXs. These channels are then merged by the predesigned $m_{l}$-channel TMUXs and the delays caused by the TMUXs are compensated in other branches.

The operation of the dynamic recombination algorithm is illustrated in Fig. 9 and Fig. 10 for two input signals at two different time-slots. Each input signal consists of 100 samples and they are determined from their frequency spectrum. The frequency response of the signal at the first time-slot is shown in Fig. 9(b) with the following parameters: $\omega_{p_{1}}=0.04 \times(2 \pi)$, $\omega_{s_{1}}=0.06 \times(2 \pi), \omega_{s_{2}}=0.19 \times(2 \pi), \omega_{p_{2}}=0.215 \times(2 \pi)$, $\omega_{p_{3}}=0.2675 \times(2 \pi)$ and $\omega_{s_{3}}=0.295 \times(2 \pi)$. Here, $\omega_{p_{i}}$ and $\omega_{s_{i}}$ are, respectively, the passband and stopband cutoff frequencies for the $i$ th transition band, $i=1,2,3$. In the passband, the signal is assumed to be linear-phase with a magnitude of one, and the passband and stopband ripples are the same. This simplification allows us to determine its time-domain value by treating it as a filter design problem (filter length $N=100$ ) having the above frequency-domain specification, and it can be solved using the REMEZ algorithm in MATLAB. Fig. 9(a) and (b) show the input signal and its spectrum in the first time-slot. Using the dynamic recombination algorithm, we find that the analysis filters $H_{0} \sim H_{3}$ and $H_{12} \sim H_{18}$ of the 32-channel uniform FB are the channels to be merged, which corresponds to the two major frequency components in Fig. 9(b). Here, we assume that the threshold is $T=-40 \mathrm{~dB}$. A 4-channel and a 7-channel predesigned TMUXs are employed to partially reconstruct these components in order to improve their time-resolution for further processing. The frequency responses of the 32-channel uniform CMFB and the 4- and 7-channel recombination CMFBs are shown in Fig. 9(g), (e), and (f), respectively. The merged outputs of channels $H_{0} \sim H_{3}$ and $H_{12} \sim H_{18}$ 


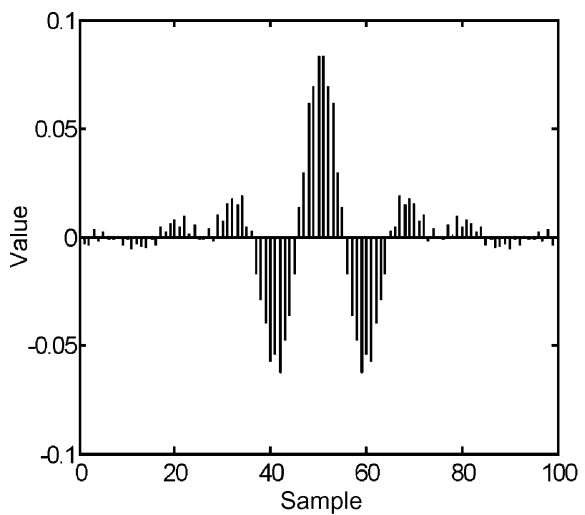

(a)

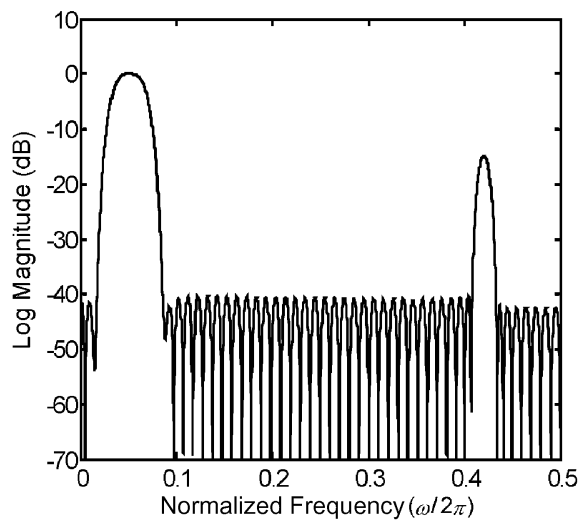

(b)

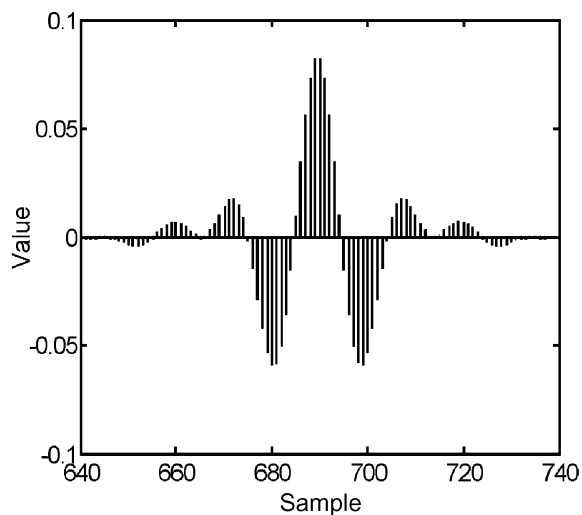

(c)

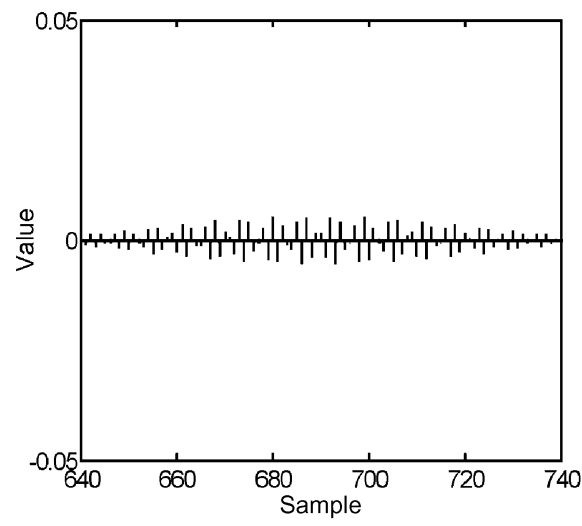

(d)

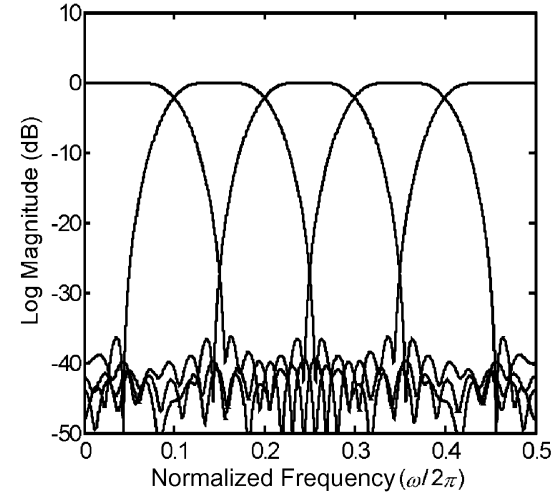

(e)

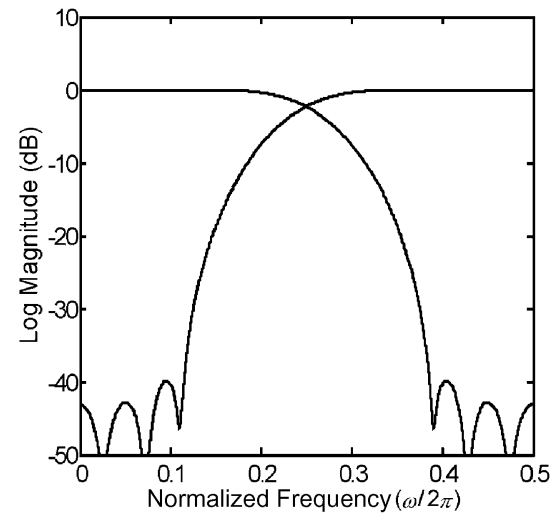

(t)

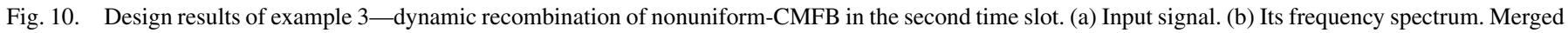

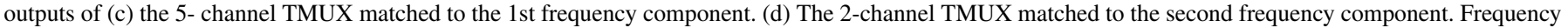
responses of the (e) 5-channel and (f) 2-channel CMFBs.

are shown, respectively, in Fig. 9(c) and (d). It can be seen that the two components are well separated by the adaptive $\mathrm{RN}-\mathrm{CMFB}$, as compared with the original 32-channel CMFB. The input signal and its spectrum at the second time-slot are shown in Fig. 10(a) and (b), respectively. Using the dynamic recombination algorithm, two predesigned 5- and 2-channel TMUXs are employed respectively to combine the outputs from $H_{1}$ to $H_{5}$, and $H_{26}$ to $H_{27}$ of the 32-channel uniform CMFB so as to better match the signal characteristics. The 5-channel and 2-channel recombination CMFBs are shown in Fig. 10(e) and (f), respectively. Fig. 10(c) and (d) shows the corresponding merged outputs. The signals are now better resolved than the uniform FB (due to page limitation, the original subband signals are omitted). It demonstrates that the proposed dynamic recombination $\mathrm{RN}-\mathrm{CMFBs}$ offer more flexibility in resolving and processing signals at adjustable time-frequency resolution than uniform FBs. It should be pointed out that, because the input signal is first decimated to a much lower rate, the arithmetic complexity of the recombination system is rather low as indicated in the next subsection.

\section{Design and Arithmetic Complexities}

We now briefly analyze the design and arithmetic complexities of the RN-CMFB using different realization methods. For comparison purpose, the complexity of the pseudo-PR CMFB in [16], which employs the direct structure in Fig. 2, is also analyzed. The result so derived was used in calculating the MPUs and APUs in Table I. Notice that although the analysis filters in [16] are derived by modulating the prototype filters with cosine modulation, they do not possess the efficient implementation structure of conventional uniform CMFBs. Therefore, it has to be implemented using the direct structure. The arithmetic complexities of the two systems are analyzed as follows:

Direct structure: Consider the polyphase implementation of the direct structure in Fig. 2. If the polyphase decomposition in Fig. 11(a) is employed, where $E_{l, j}\left(j=0,1, \cdots, q_{l}-1\right)$ are the polyphase components of $H_{l}(\omega)$, the cost is, respectively: $N_{l} p_{l} / q_{l}$ MPUs and $\left(N_{l}-1\right) p_{l} / q_{l}$ APUs, where $N_{l}$ is the filter length of $H_{l}(\omega)$. The total complexity is $\sum_{l=0}^{L-1}\left(N_{l} p_{l} / q_{l}\right)$ MPUs and $\sum_{l=0}^{L-1}\left(N_{l}-1\right) p_{l} / q_{l}$ APUs. If the polyphase decomposition is not employed, i.e., direct-implementation of $H_{l}(\omega)$, it will increase to $\sum_{l=0}^{L-1} N_{l} p_{l}$ MPUs and $\sum_{l=0}^{L-1}\left(N_{l}-1\right) p_{l}$ APUs.

Recombination structure: The polyphase implementation in Fig. 11(b) is employed for the $l$ th branch of the RN-CMFB in Fig. 1, where $E_{k}(k=0,1, \cdots, M-1)$ and $R_{l, i}(i=$ $\left.0,1, \cdots, m_{l}-1\right)$ are, respectively, the polyphase component of the prototype filters $H(\omega)$ and $G_{l}(\omega) .5$ The cost of the system

${ }^{5}$ For more details on the implementation of paraunitary CMFBs, see [13]. For simplicity, we consider only the polyphase implementation of $H(\omega)$ instead of the lossless lattice. 


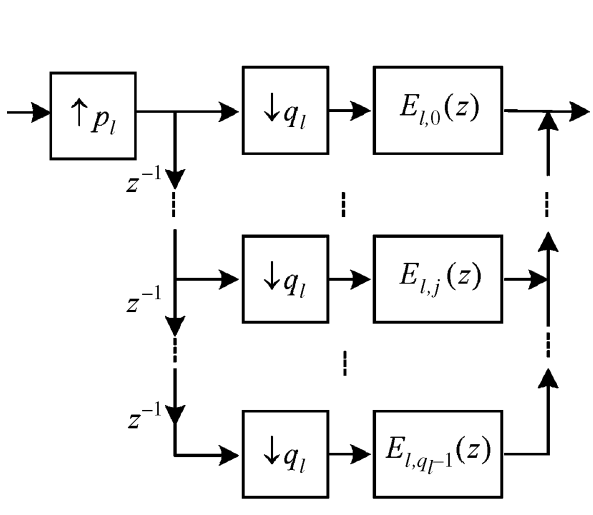

(a)

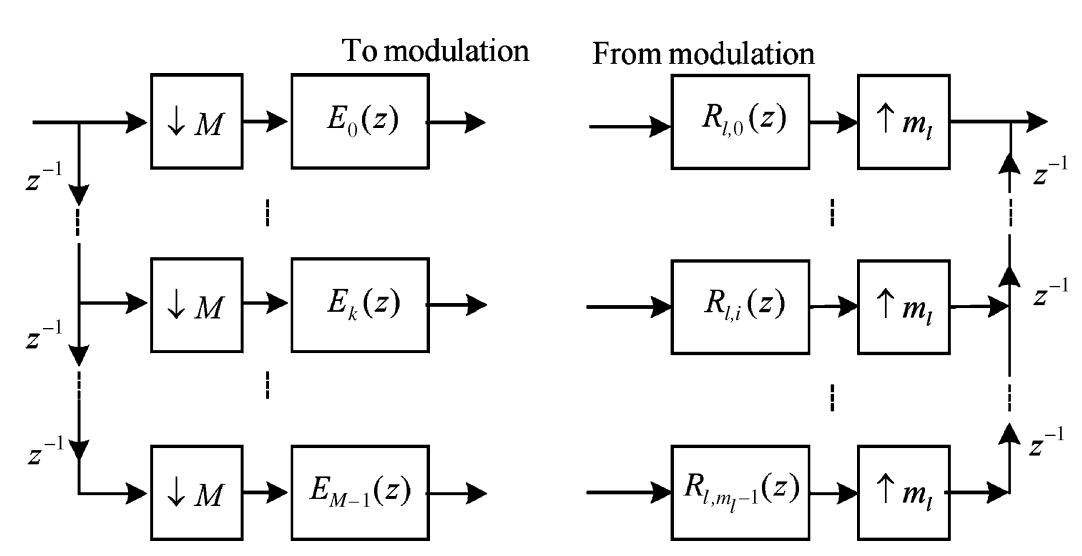

(b)

Fig. 11. (a) Polyphase component implementation of direct structure. (b) Polyphase component implementation of the RN-CMFB.

is $(1 / M)\left(N_{M}+\sum_{l=0}^{L-1} N_{m_{l}}\right)$ MPUs and $(1 / M)\left(N_{M}-1+\right.$ $\left.\sum_{l=0}^{L-1}\left(N_{m_{l}}-1\right)\right)$ APUs. Moreover, the cost of modulation should be included. Note, although there are $M$ filter outputs in the original CMFB, its complexity excluding the modulation is only $N_{M} / M$ MPUs and $\left(N_{M}-1\right) / M$ APUs. This is equal to a single $M$-fold decimator with filter length $N_{M}$. As the stopband attenuation and transition bandwidth of an FIR filter depend on the filter length, the arithmetic complexity of CMFBs is almost independent of the channel number for the same stopband attenuation and transition bandwidth. Similar argument applies to the CMFB-based TMUXs. This explains the low implementation complexity of our RN-CMFBs, despite the apparent impression that more channels have to be realized. It can be seen that the proposed RN-CMFBs possess a very low implementation complexity, thanks to the efficient CMFBs and the interpolation-filter structure used in RNFB.

\section{CONCLuSiON}

The theory and design of a class of RNFBs with more flexible sampling factors is presented. The spurious response and spectral inversion problems are analyzed and a matching condition is proposed for the efficient design of RNFB using cosine modulation. Design results show that high quality and low complexity nonuniform CMFBs can be obtained by the proposed method while the PR property of the FBs is maintained. Another advantage of the proposed method is that the FBs can be designed separately so that adaptive merging can be performed to provide variable time-frequency resolution.

\section{APPENDIX}

\section{MATCHING CONDITION FOR ODD VALUE OF $r_{l}$}

When $r_{l}$ is odd, spectral inversion may appear. To overcome this problem, we can modulate the output of the channels to be merged by the sequence $\left\{(-1)^{n}\right\}$ [3]-[5], [14]. Accordingly, $Y_{l}(\omega)$ in $(7), Y_{l}(\omega)$ in $(8), D_{1}^{(v)}(\omega)$ and $D_{2}^{(v)}(\omega)$ in (15) are changed to

$$
\begin{gathered}
Y_{l}(\omega)=\frac{1}{M} \sum_{v=0}^{M-1} A_{v}(\omega) X\left(\frac{m_{l} \omega-2 \pi v+\pi}{M}\right), \\
v=0,1, \cdots, M-1
\end{gathered}
$$

$$
\begin{aligned}
A_{v}(\omega)= & \sum_{i=0}^{m_{l}-1} G_{l, i}(\omega) \bar{H}_{r_{l}+i}\left(\omega-\frac{2 \pi v-\pi}{m_{l}}\right) \\
= & e^{-j \omega} \sum_{i=0}^{m_{l}-1} G_{l, i_{-} F B}(\omega) \bar{H}_{r_{l}+i}\left(\omega-\frac{2 \pi v-\pi}{m_{l}}\right) \\
= & e^{-j \omega} \sum_{j=1}^{4} D_{j}^{(v)}(\omega) \\
D_{1}^{(v)}(\omega)= & \frac{1}{4} \sum_{i=0}^{m_{l}-1} e^{j\left(\theta_{r_{l}+i}+\xi_{l, i}\right)} \\
& \times \bar{H}_{r_{l}+i}^{+}\left(\omega-\frac{2 \pi v-\pi}{m_{l}}\right) G_{l, i}^{+}(\omega) \\
D_{2}^{(v)}(\omega)= & \frac{1}{4} \sum_{i=0}^{m_{l}-1} e^{j\left(\theta_{r_{l}+i}+\xi_{l, i}\right)} \\
& \times \bar{H}_{r_{l}+i}^{+}\left(\omega-\frac{2 \pi v-\pi}{m_{l}}\right) G_{l, i}^{-}(\omega)
\end{aligned}
$$

We only consider $D_{1}^{(v)}(\omega)$ and $D_{2}^{(v)}(\omega)$ because $D_{3}^{(v)}(\omega)$ and $D_{4}^{(v)}(\omega)$ are their conjugates.

Supports of $\bar{H}_{r_{l}+i}(\omega)$ and $G_{l, i}(\omega)$ : Due to the modulation of $\left\{(-1)^{n}\right\}$, the frequency support of $\bar{H}_{r_{l}+i}\left(\omega-\left(2 \pi v-\pi / m_{l}\right)\right)$ is changed from (17) to

$$
\begin{aligned}
\operatorname{Supp} & \left(\bar{H}_{r_{l}+i}\left(\omega-\frac{2 \pi v-\pi}{m_{l}}\right)\right) \\
= & \operatorname{Supp}\left(\bar{H}_{r_{l}+i}^{+}\left(\omega-\frac{2 \pi v-\pi}{m_{l}}\right)\right) \\
& \cup \operatorname{Supp}\left(\bar{H}_{r_{l}+i}^{-}\left(\omega-\frac{2 \pi v-\pi}{m_{l}}\right)\right) \\
= & x_{v, r_{l}+i}^{+} \cup x_{v, r_{l}+i}^{-},
\end{aligned}
$$

where

$$
\begin{aligned}
x_{v, r_{l}+i}^{+} & =\left[\frac{\pi}{m_{l}}\left(2 v+\left(r_{l}+i\right)-\frac{3}{2}\right), \frac{\pi}{m_{l}}\left(2 v+\left(r_{l}+i\right)+\frac{1}{2}\right)\right] \\
& =\left[s_{v, r_{l}+i}^{+}, t_{v, r_{l}+i}^{+}\right], \\
x_{v, r_{l}+i}^{-} & =\left[\frac{\pi}{m_{l}}\left(2 v-\left(r_{l}+i\right)-\frac{5}{2}\right), \frac{\pi}{m_{l}}\left(2 v-\left(r_{l}+i\right)-\frac{1}{2}\right)\right] \\
& =\left[s_{v, r_{l}+i}^{-}, t_{v, r_{l}+i}^{-}\right] .
\end{aligned}
$$


$\operatorname{Supp}\left(G_{l, i_{-} F B}(\omega)\right)$ is identical to the case when $r_{l}$ is even [(19) and (20)].

Support of $D_{1}^{(v)}(\omega)$ : The starting location of $D_{1}^{(v)}(\omega)$ in (A3) is determined by

$$
\begin{aligned}
s_{v, r_{l}+i}^{+}-u_{q, i}^{+}= & \frac{\pi}{m_{l}}\left(2 v+\left(r_{l}+i\right)-\frac{3}{2}\right) \\
& -\frac{\pi}{m_{l}}\left(2 m_{l} q+i-\frac{1}{2}\right) \\
= & \left(v-\frac{1}{2}+\frac{r_{l}}{2}-m_{l} q\right) I \\
= & \left(v+r_{l}^{\prime}-m_{l} q\right) I
\end{aligned}
$$

where $r_{l}=2 r_{l}^{\prime}+1$, since $r_{l}$ is odd. From (A5), we can see that

$$
\begin{cases}{\left[s_{v, r_{l}+i}^{+}, t_{v, r_{l}+i}^{+}\right]=\left[u_{q, i}^{+}, v_{q, i}^{+}\right]} & \text {if } v=m_{l} q-r_{l}^{\prime} \\ {\left[s_{v, r_{l}+i}^{+}, t_{v, r_{l}+i}^{+}\right] \cap\left[u_{q, i}^{+}, v_{q, i}^{+}\right]=\phi,} & \text { if } v \neq m_{l} q-r_{l}^{\prime}\end{cases}
$$

For the $v=m_{l} q-r_{l}^{\prime}$ case, we have

$$
\begin{aligned}
& \operatorname{Supp}\left(\bar{H}_{r_{l}+i}^{+}\left(\omega-\frac{2 \pi v-\pi}{m_{l}}\right) G_{l, i}^{+}(\omega)\right) \\
& =\left[s_{v, r_{l}+i}^{+}, t_{v, r_{l}+i}^{+}\right] \\
& =\left[\frac{\pi}{m_{l}}\left(2 v+r_{l}+i-\frac{3}{2}\right), \frac{\pi}{m_{l}}\left(2 v+r_{l}+i+\frac{1}{2}\right)\right] .
\end{aligned}
$$

Therefore

$$
\begin{cases}\operatorname{Supp}\left(D_{1}^{(v)}\right) & \\ =\left[\frac{\pi}{m_{l}}\left(2 v+r_{l}-\frac{3}{2}\right), \frac{\pi}{m_{l}}\left(2 v+r_{l}+m_{l}-\frac{1}{2}\right)\right], & \text { if } v=m_{l} q-r_{l}^{\prime} \\ \operatorname{Supp}\left(D_{1}^{(v)}\right)=\phi, & \text { if } v \neq m_{l} q-r_{l}^{\prime}\end{cases}
$$

and it belongs to the desired support of an ideal decimator.

Support of $D_{2}^{(v)}(\omega)$ : The starting location of $D_{2}^{(v)}(\omega)$ in (A3) is

$$
\begin{aligned}
s_{v, r_{l}+i}^{+}-u_{q, i}^{-}= & \frac{\pi}{m_{l}}\left(2 v+\left(r_{l}+i\right)-\frac{3}{2}\right) \\
& -\frac{\pi}{m_{l}}\left(2 m_{l} q-i-\frac{3}{2}\right) \\
= & \left(v-m_{l} q+r_{l}^{\prime}+i+\frac{1}{2}\right) I .
\end{aligned}
$$

Therefore, $\left[s_{v, r_{l}+i}^{+}, t_{v, r_{l}+i}^{+}\right] \cap\left[u_{q, i}^{-}, v_{q, i}^{-}\right] \neq \phi$ if one of the following equations holds

$$
\begin{aligned}
& v-m_{l} q+r_{l}^{\prime}+i=0 \\
& v-m_{l} q+r_{l}^{\prime}+i=-1 .
\end{aligned}
$$

When (A9) holds, it can be derived from (A8) that $s_{v, r_{l}+i}^{+}-$ $u_{q, i}^{-}=I / 2$. Then

$$
\begin{aligned}
& {\left[s_{v, r_{l}+i}^{+}, t_{v, r_{l}+i}^{+}\right] \cap\left[u_{q, i}^{-}, v_{q, i}^{-}\right]} \\
& \quad=\left[s_{v, r_{l}+i}^{+}, v_{q, i}^{-}\right] \\
& \quad=F_{v, i}^{+} \\
& \quad=\left[\frac{\pi}{m_{l}}\left(2 v+r_{l}+i-\frac{3}{2}\right), \frac{\pi}{m_{l}}\left(2 m_{l} q-i+\frac{1}{2}\right)\right] .
\end{aligned}
$$

When (A10) holds, we have $s_{v, r_{l}+i}^{+}-u_{q, i}^{-}=-I / 2$, and

$$
\begin{aligned}
& {\left[s_{v, r_{l}+i}^{+}, t_{v, r_{l}+i}^{+}\right] \cap\left[u_{q, i}^{-}, v_{q, i}^{-}\right]} \\
& \quad=\left[u_{q, i}^{-}, t_{v, r_{l}+i}^{+}\right] \\
& \quad=F_{v, i}^{-} \\
& \quad=\left[\frac{\pi}{m_{l}}\left(2 m_{l} q-i-\frac{3}{2}\right), \frac{\pi}{m_{l}}\left(2 v+r_{l}+i+\frac{1}{2}\right)\right] .
\end{aligned}
$$

Hence

$$
\begin{aligned}
D_{2}^{(v)}(\omega)= & \frac{1}{4} \sum_{i=0}^{m_{l}-1} e^{j\left(\theta_{r_{l}+i}-\xi_{l, i}\right)} \\
& \times \bar{H}_{r_{l}+i}^{+}\left(\omega-\frac{2 \pi v-\pi}{m_{l}}\right) G_{l, i}^{-}(\omega) \\
= & \frac{1}{4} e^{j\left(\theta_{r_{l}-\xi_{l, 0}}\right)} \bar{H}_{r_{l}}^{+}\left(\omega-\frac{2 \pi v-\pi}{m_{l}}\right) G_{l, 0}^{-}(\omega) E_{F_{v, 0}^{+}} \\
& +\frac{1}{4} \sum_{i=0}^{m_{l}-2}\left(e^{j\left(\theta_{r_{l}+i+1}-\xi_{l, i+1}\right)}\right. \\
& \times \bar{H}_{r_{l}+i+1}^{+}\left(\omega-\frac{2 \pi v-\pi}{m_{l}}\right) \\
& \times \frac{1}{4} e^{j\left(\theta_{r_{l}+m_{l}-1-\xi_{l, m_{l}-1}}^{-}\right.}(\omega) E_{F_{v, i+1}^{+}}+e^{j\left(\theta_{r_{l}+i-\xi_{l, i}}\right)} \\
& \times \bar{H}_{r_{l}+m_{l}-1}^{+}\left(\omega \bar{H}_{r_{l}+i}^{+}\left(\omega-\frac{2 \pi v-\pi}{m_{l}}\right) G_{l, i}^{-}(\omega) E_{F_{v, i}^{-}}\right) \\
\left.m_{l}\right) G_{l, m_{l}-1}^{-} E_{F_{v, m_{l}-1}^{-}} &
\end{aligned}
$$

Again, it can be shown that $E_{F_{v, i+1}^{+}}=E_{F_{v, i}^{-}}$, and the cross-term in (A13) is reduced to zero if the matching condition in (32) and (33) is satisfied.

\section{REFERENCES}

[1] P. P. Vaidyanathan, "Multirate digital filters, filter banks, polyphase network, and applications: A tutorial," Proc. IEEE, vol. 78, no. 1, pp. 56-93, Jan. 1990.

[2] T. Blu, "An iterated rational filter bank for audio coding time-frequency and time-scale analysis," in Proc. IEEE Int. Symp. Signal Process., Jun. 1996, pp. 81-84.

[3] R. V. Cox, "The design of uniformly and nonuniformly spaced pseudoquadrature mirror filters," IEEE Trans. Acoust. Speech Signal Process., vol. ASSP-24, no. 5, pp. 1090-1096, Oct. 1986.

[4] J. Kovacevic and M. Vetterli, "Perfect reconstruction filter bank with rational sampling factors," IEEE Trans. Signal Process., vol. 41, no. 6, pp. 2047-2066, Jun. 1993.

[5] S. C. Chan, X. M. Xie, and T. I. Yuk, "Theory and design of a class of cosine-modulated nonuniform filter banks," in Proc. IEEE ICASSP'00, vol. 1, 2000, pp. 504-507.

[6] X. M. Xie, S. C. Chan, and T. I. Yuk, "A class of biorthogonal nonuniform cosine-modulated filter banks with lower system delay," in Proc. IEEE ISCAS'01, vol. 2, 2001, pp. 25-28.

[7] P. Q. Hoang and P. P. Vaidyanathan, "Nonuniform multirate filter banks: theory and design," in Proc. IEEE ISCAS'89, 1989, pp. 371-374.

[8] S. Akkarakaran and P. P. Vaidyanathan, "New results and open problems on nonuniform filter banks," in Proc. IEEE ICASSP'99, vol. 3, 1999, pp. 1501-1504.

[9] R. L. Queiroz, "Uniform filter banks with nonuniform bands: post-processing design," in Proc. IEEE ICASSP'98, vol. 3, 1998, pp. 12-15.

[10] K. Nayebi, T. P. Barnwell III, and M. J. T. Smith, "Nonuniform filter banks: a reconstruction and design theory," IEEE Trans. Signal Process., vol. 41, no. 3, pp. 1114-1127, Mar. 1993. 
[11] J. Princen, "The design of nonuniform modulated filterbanks," IEEE Trans. Signal Process., vol. 43, no. 11, pp. 2550-2560, Nov. 1995.

[12] R. D. Koilpillai and P. P. Vaidyanathan, "Cosine-modulated FIR filter banks satisfying perfect reconstruction," IEEE Trans. Signal Process., vol. 40, no. 4, pp. 770-783, Apr. 1992.

[13] P. P. Vaidyanathan, Multirate Systems and Filter Banks. Englewood Cliffs, NJ: Prentice-Hall, 1992

[14] X. M. Xie and S. C. Chan, "On the application of modulation in designing perfect-reconstruction recombination nonuniform filter banks," in Proc. IEEE ICASSP(student paper forum), 2003.

[15] R. D. Koilpillai, T. Q. Nguyen, and P. P. Vaidyanathan, "Some results in the theory of crosstalk-free transmultiplexers," IEEE Trans. Signal Process., vol. 39, no. 10, pp. 2174-2183, Oct. 1991.

[16] F. Argenti, B. Brogelli, and E. Del Re, "Design of pseudo-QMF banks with rational sampling factors using several prototype filters," IEEE Trans. Signal Process., vol. 46, no. 6, pp. 1709-1715, Jun. 1998

[17] X. M. Xie, S. C. Chan, and T. I. Yuk, "A class of perfect-reconstruction nonuniform cosine-modulated filter banks with dynamic recombination," in Proc. Eusipco'02, vol. 2, 2002, pp. 549-552.

[18] T. W. Chen, L. Qiu, and E. W. Bai, "General multirate building structures with application to nonuniform filter banks," IEEE Trans. Circuits Syst. II, Analog Digit. Signal Process.,., vol. 45, no. 8, pp. 948-958, Aug. 1998.

[19] M. Vetterli and J. Kovacevic, Wavelets and Subband Coding. Englewood Cliffs, NJ: Prentice-Hall, 1995.

[20] A. N. Akansu and R. A. Haddad, Multiresolution Signal Decomposition: Transforms, Subbands, and Wavelets. San Diego, CA: Academic, 2001

[21] N. J. Fliege, Multirate Digital Signal Processing: Multirate Systems, Filter Banks, Wavelets. Chichester, U.K.: Wiley, 1994.

[22] H. S. Malvar, Signal Processing With Lapped Transforms. Boston, MA: Artech House, 1992.

[23] J. Li, T. Q. Nguyen, and S. Tantaratana, "A simple design method for near-perfect reconstruction nonuniform filter banks," IEEE Trans. Signal Process., vol. 45, no. 8, pp. 2105-2109, Aug. 1997.

[24] S. Wada, "Design of nonuniform division multirate FIR filter banks," IEEE Trans. Circuits Syst. II, Analog Digit. Signal Process., vol. 42, no. 2, pp. 115-121, Feb. 1995.

[25] S. C. Chan and X. M. Xie, "Biorthogonal recombination nonuniform cosine-modulated filter banks and multiplierless realizations," in $J$. VLSI Signal Process., to be published.

[26] P. N. Heller, T. Karp, and T. Q. Nguyen, "A general formulation of modulated filter banks," IEEE Trans. Signal Process., vol. 47, no. 4, pp. 986-1002, Apr. 1999

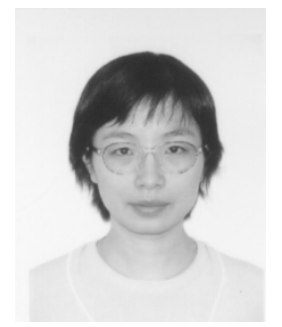

X. M. Xie received the M.S. degree in electronic engineering from Xidian University, China, in 1996 , and the Ph.D. degree in electrical and electronic engineering from the University of Hong Kong in 2004.

She is now with the School of Electronic Engineering, Xidian University. Her research interests are in digital signal processing, multirate filter bank, and wavelet transform.

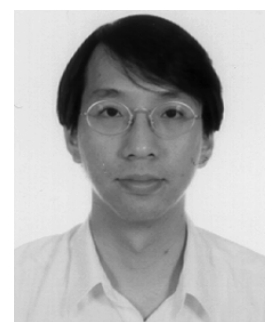

S. C. Chan received the B.Sc. (Eng.) and Ph.D. degrees in electrical engineering from the University of Hong Kong, Hong Kong, in 1986 and 1992, respectively.

He joined City Polytechnic of Hong Kong in 1990 as an Assistant Lecturer and later as a University Lecturer. Since 1994, he has been with the Department of Electrical and Electronic Engineering, the University of Hong Kong, Hong Kong, and is now an Associate Professor. He was a visiting researcher with Microsoft Corporation, Redmond, and Microsoft China in 1998 and 1999, respectively. His research interests include fast transform algorithms, filter design and realization, multirate signal processing, communications signal processing, and image-based rendering.

Dr. Chan is currently a member of the Digital Signal Processing Technical Committee of the IEEE Circuits and Systems Society. He was Chairman of the IEEE Hong Kong Chapter of Signal Processing from 2000 to 2002.

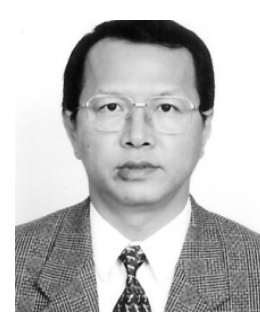

T. I. Yuk received the B.S. degree from Iowa State University, Ames, in 1978, and the M.S. and Ph.D. degrees from Arizona State University, Tucson, in 1980 and 1986 respectively.

Since 1986, he has been a Lecturer at the University of Hong Kong, Hong Kong. His current research interests include performance analysis and coding of MIMO systems and communication signal processing. 